\title{
CLASSIFICATION OF SEMISIMPLE ALGEBRAIC MONOIDS
}

\author{
BY
}

LEX E. RENNER

\begin{abstract}
Let $X$ be a semisimple algebraic monoid with unit group $G$. Associated with $E$ is its polyhedral root system $(X, \Phi, C)$, where $X=X(T)$ is the character group of the maximal torus $T \subseteq G, \Phi \subseteq X(T)$ is the set of roots, and $C=X(\bar{T})$ is the character monoid of $\bar{T} \subseteq E$ (Zariski closure).

The correspondence $E \rightarrow(X, \Phi, C)$ is a complete and discriminating invariant of the semisimple monoid $E$, extending the well-known classification of semisimple groups. In establishing this result, monoids with preassigned root data are first constructed from linear representations of $G$. That done, we then show that any other semisimple monoid must be isomorphic to one of those constructed. To do this we devise an extension principle based on a monoid analogue of the big cell construction of algebraic group theory. This, ultimately, yields the desired conclusions.
\end{abstract}

Consider the classification problem for semisimple, algebraic monoids over the algebraically closed field $k$.

What sort of problem is this? First the definition:

A semisimple, algebraic monoid is an irreducible, affine, algebraic variety $E$, defined over $k$ together with an associative morphism $m: E \times E \rightarrow E$ and a two-sided unit $1 \in E$ for $m$. We assume further that $E$ has a 0 , the unit group $G$ (which is always linear, algebraic and dense in $E$ ), is reductive (e.g. $\mathrm{Gl}_{n}(k)$ ), $\operatorname{dim} Z G=1$ and $E$ is a normal variety.

The problem then, presents us with two familiar objects. Let $T \subseteq G$ be a maximal torus. Then

$Z=\bar{T} \subseteq E$ (Zariski closure) is an affine, torus embedding and

$G \subseteq E$ is a reductive, algebraic group.

Torus embeddings have been introduced by Demazure in [6] in his study of Cremona groups, and are classified numerically using rational, polyhedral cones [14].

On the other hand, reductive groups have been studied, at least in principle, since the nineteenth century; their classification in the modern sense being achieved largely by Chevalley [4]. That numerical classification uses the now familiar root systems [11, Chapter 3] of Killing that were introduced by him [15] in his penetrating formulation of the classification (E. Cartan's!) of semisimple Lie algebras.

Thus, in the classification of semisimple monoids we are compelled to consider the root system $(X, \Phi)=(X(T), \Phi(T))$ of $G$, and the polyhedral cone $C=X(Z) \subseteq X(T)$ of $T \subseteq Z$. The two objects are canonically related by the Weyl group action on $X$, which leaves $C$ stable.

Received by the editors November 16, 1984.

1980 Mathematics Subject Classification. Primary 14M99; Secondary 20M99.

Key words and phrases. Semisimple monoid, polyhedral root system. 
C(I) $\cap \mathrm{H}$

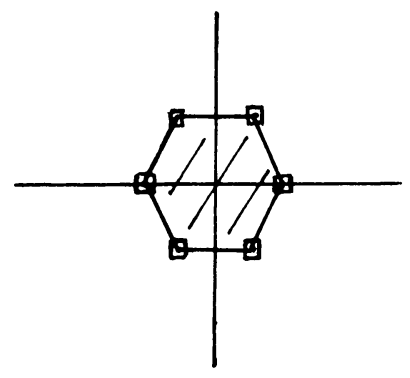

$\mathrm{C}(2) \cap \mathrm{H}$

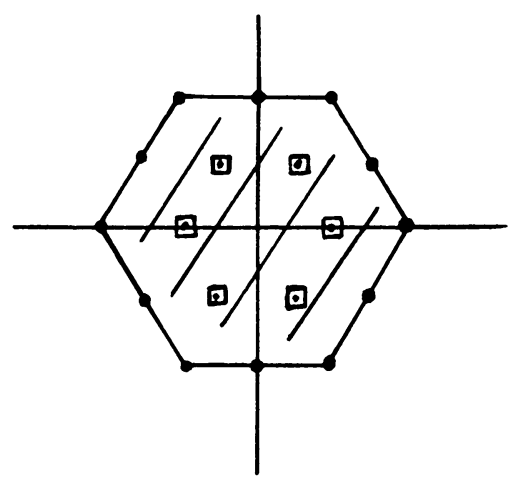

FIGURE 1

$(X, \Phi, C)$ is the polyhedral root system of $E$, and as such it belongs to an axiomatically defined class of numerical objects (3.6).

The main result of this paper (6.5) asserts that the correspondence $E \rightarrow(X, \Phi, C)$ is a complete and discriminating invariant of the semisimple, algebraic monoid $E$.

What then is to be learned from such a classification and what important properties of semisimple groups are most involved?

First of all, the reader is encouraged to think of the theory of semisimple monoids as a universal, geometric model for the representation theory of the underlying semisimple group $G_{0}=(G, G)$. These monoids provide the fundamental link between linear representations of $G_{0}$ and numerical and geometric structures associated with certain types of $G_{0}$-actions. The actions we have in mind are of the form

$$
\begin{gathered}
\varphi: G_{0} \times W \rightarrow W, \\
W=\operatorname{Spec}(\Gamma), \quad \Gamma=\bigoplus_{n \geqslant 0} \Gamma_{n}, \quad \Gamma_{0}=k .
\end{gathered}
$$

In this case we let $E(\Gamma)=$ normalization of $\overline{G_{0} \cdot k^{*}} \subseteq \operatorname{End}(\Gamma)$, where $k^{*} \rightarrow \operatorname{Aut}(\Gamma)$ is the tautological action. We assume here that $\varphi$ preserves this action.

Such actions arise in geometric invariant theory whenever one linearizes, with an ample line bundle, the $G_{0}$-action on a projective variety [17, Chapter 1].

So we think of $Z=\operatorname{Proj}(\Gamma)$ and $\mu: G_{0} \times Z \rightarrow Z$, induced from $\varphi$, as being kept fixed, and $\Gamma$ as varying within these constraints. We may then obtain a canonical correspondence

$$
\Gamma \rightarrow(X, \Phi, C(\Gamma)),
$$

where $(X, \Phi, C(\Gamma))$ is the polyhedral root system of $E(\Gamma)$.

For a simple illustration take $Z=\mathbf{P}^{8}=\operatorname{Proj}\left(k\left[x_{11}, \ldots, x_{33}\right]\right), G_{0}=\mathrm{PGl}_{3}(k)$ and $G_{0} \times Z \rightarrow Z$, the action induced from the adjoint representation $\mathrm{Gl}_{3}(k) \rightarrow \mathrm{Gl}_{9}(k)$. $k\left[x_{11}, \ldots, x_{33}\right]=\bigoplus_{n \geqslant 0} \Gamma_{n}=\Gamma$ is graded in the usual way and we let

$$
\Gamma(m)=\bigoplus_{n \geqslant 0} \Gamma_{m n}
$$




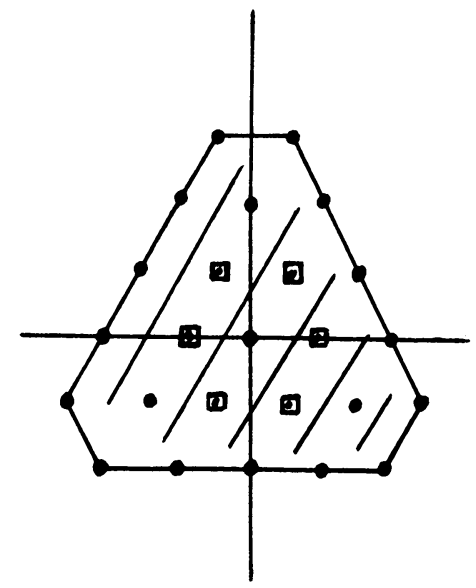

Figure 2

Thus, we obtain in this case,

$$
\Gamma(m) \rightarrow(X, \Phi, C(\Gamma(m))) .
$$

We can reveal $C(m)=C(\Gamma(m))$ most easily by taking a hyperplane section as in Figure 1. Here $X=X_{0} \oplus \mathbf{Z}\left(=X\left(T \cap G_{0}\right) \oplus X(Z G)\right), C(m) \subseteq X, H=\{(\chi, l) \in$ $X \mid l=1\}$ and $\boxminus=(\chi, 1)$ indicates that $\chi$ is a root.

In more complicated examples we would obtain Figure 2, as well as the above configurations in Figure 1, for some instances of $\Gamma$.

I like to think of $\Gamma \rightarrow(X, \Phi, C(\Gamma))$ as a "dynamic, numerical picture" of the ample, $G_{0}$-linear line bundles on $Z$. In general, $C(\Gamma)$ can vary considerably, but the configuration of faces of $C(\Gamma)$ and the angles between them will remain constant.

We cannot pursue this discussion any further, so instead we must urge the reader to consider these monoids as a natural and geometric object associated with semisimple groups; a theory that will, at the very least, add a new and exciting dimension to the geometric theory of transformation groups.

1. Introduction. Chevalley's classification of semisimple groups has been significantly refined and simplified by Borel [2]. Our basic reference for this material will be Humphreys' book [12] (see also [33]). In particular, no mention will be made of rationality problems (which will ultimately be studied using the techniques of [13]).

The theory of torus embeddings [14] is the other major, geometric ingredient in our theory. However, we shall need only results from the elementary, affine theory. Chapter 1 of [14] and Putcha's paper [20] should provide adequate background for the reader.

Putcha [19-26] initiated the study of algebraic semigroups publicly in 1980 and has recorded many useful results from the semigroup point of view (Green's relations, conjugacy classes of idempotents, etc.). The cumulative effect of Putcha's work on mine is quite significant. It is certain that the results of this paper would not 
have been possible without the results of [21-24]. Much of that work is directed at the problem of interpreting notions from abstract semigroup theory on an algebraic monoid.

In [21] Green's relations are studied on an irreducible algebraic monoid. A key result is that idempotents $e$ and $f$ in the same $\mathscr{f}$-class $(e \in G f G)$ are actually conjugate. This is needed here in (8.3.3) and is used throughout the theory of algebraic monoids.

The underlying theme of [22] is "How can the reductive, algebraic group $G$ be studied using an algebraic monoid $E$ with 0 and unit group $G$ ?" The main results imply that every Borel subgroup of $G$ is the left centralizer of a maximal chain of indempotents $\Gamma \subseteq E$,

$$
B=\{g \in G \mid e g=e g e \text { for all } e \in \Gamma\} .
$$

The notion of a cross-section lattice $\Lambda$ (see $\S 4$ ), is introduced there for the first time.

In [24] the basic properties of these $\Lambda$ are recorded and in [26] the striking result

$$
\begin{gathered}
\{B \mid T \subseteq B\} \stackrel{\cong}{\rightarrow}\{\Lambda \subseteq I \bar{T} \mid \Lambda \text { is a cross-section lattice }\}, \\
B \rightarrow\left\{e \in \bar{T} \mid e=e^{2} \text { and } e b=e b e \text { for all } b \in B\right\}
\end{gathered}
$$

is obtained. This helps us arrive at a very appealing generalization (4.4) of Chevalley's big cell construction, which is perhaps our most powerful technical result.

In [23] Putcha proves that any reductive monoid in characteristic 0 is regular using Weyl's Theorem on complete reducibility, and some of his own clever "elementary" calculations. In [29] I have generalized these results to any characteristic, but the basic idea behind Putcha's arguments are still used even though Weyl's theorem does not hold in general.

The usefulness of the result should not be underestimated. For example, if $\varphi$ : $E \rightarrow E^{\prime}$ is a morphism of algebraic monoids, $0 \in E$ and $E$ is reductive, then $\varphi$ is finite if and only if $\varphi \mid \bar{T}$ is finite. Thus, on a reductive monoid, finiteness can be detected by the polyhedral root system. If further, $\varphi$ is birational and $E^{\prime}$ normal, then $\varphi$ is an isomorphism. This kind of argument is used in (6.3).

The classification problem of semisimple monoids was first studied by myself in [30] where a complete, numerical classification was obtained for the semisimple rank one case. The main result of [30] yields a canonical one-to-one correspondence

$$
\mathscr{E}(G) \cong \mathbf{Q}^{+},
$$

where $G=\mathrm{Gl}_{2}(k), \mathrm{Sl}_{2}(k) \times k^{*}$ or $\mathrm{PGl}_{2}(k) \times k^{*}$ and $\mathscr{E}(G)$ is the set of isomorphism classes of semisimple monoids with unit group $G$. The general case does not admit such a simple formulation, but the above correspondence is still explained, in general terms, in (8.3). Monoids of that type ( $\mathscr{J}$-irreducible) are the ones most directly related to irreducible representations of $G$.

$\$ 2$ is a recollection and summary of the background information most important for our logical developments. Other than Zariski's main theorem and a version of Nakayama's lemma, very little is needed from algebraic geometry, beyond the usual requirements for linear, algebraic groups [12, Chapter 1]; and in particular, we shall not require any new infinitesimal methods. 
In $\S 3$ we relate the highest weight vector of an irreducible representation $\rho$ : $G_{0} \rightarrow \mathrm{Gl}(V)$ to the minimal indempotents of $\overline{\rho\left(T_{0}\right) \cdot Z \mathrm{Gl}(V)}$ and find that the weight of $\rho$ is an extreme weight (in the sense of convexity) of $\Phi(\rho)$, the weights of $\left.\rho\right|_{T}$ (3.5). This is an important link between representation theory and torus embeddings, and becomes a major tool in the construction of all semisimple monoids (5.1).

$\$ 4$ contains the construction of the big cell. Starting with some results of [ 24 and 30] we construct, for each maximal idempotent $e \in \bar{T}$, a Borel subgroup $B \subseteq G$ such that

$$
T \subseteq B \quad \text { and } \quad B_{u}^{-} \times T_{e} \times B_{u} \rightarrow E, \quad(x, y, z) \rightarrow x y z,
$$

is an open embedding, where $B^{-}$is the opposite Borel subgroup and $T_{e}$ is a certain open submonoid of $\bar{T}$ with $e \in T_{e}$. This is our major tool, and is used to show that distinct, semisimple monoids have distinct polyhedral root systems (6.5).

$\$ 5$ contains the actual construction of all semisimple monoids. It is still unclear, at this point, that $(X(T), \Phi(T), X(Z))$ is actually a polyhedral root system! (Why is $Z$ normal?)

In $\$ 6$ we complete the proof of our main result (6.5). That $Z$ is normal now follows from a careful application of the big cell construction, i.e. the big cell construction is a powerful enough tool for us to construct for a given $E$ a finite birational morphism $\rho: E^{\prime} \rightarrow E$ such that $\left.\rho\right|_{Z^{\prime}}: Z^{\prime} \rightarrow Z$ is the normalization of $Z$ (6.1). Since $E$ is normal, $\rho$ and thus $\left.\rho\right|_{Z^{\prime}}$ are isomorphisms.

To aid the presentation of my main ideas I have left a few gaps in some arguments, especially in $\$ 5 . \$ 7$ contains the remaining details necessary to make the proofs of these results as stated, adequately general. We also consider some further refinements of the classification, and a short preliminary discussion of anti-involutions.

In $\S 8$ we discuss several immediate consequences of the classification theory. The first is the classification of birational morphisms between semisimple monoids to within an inner automorphism. In each case, there are but a finite number, and the enumeration is stated directly in terms of the polyhedral root systems involved and the severely restricted group $\Gamma=$ Aut $^{+}(G) \backslash \operatorname{Inn}(G)$.

The second consequence concerns opposite monoids. Associated with any monoid $E$ is the opposite monoid $E^{\mathrm{op}}$. $E^{\mathrm{op}}=E$ as an algebraic variety, but the order of multiplication in $E^{\mathrm{op}}$ is reversed; $(x \cdot y)_{\mathrm{op}}=y x$. We obtain the pleasing result that $\tau: E \cong E^{\mathrm{op}}$ for any semisimple, algebraic monoid (8.2). Furthermore, we may choose $\tau$ particularly well behaved.

Our next application concerns $\mathscr{Z}$-irreducible monoids. A semisimple monoid $E$ is $\mathscr{J}$-irreducible if it has a unique minimal $\mathscr{J}$-class; equivalently, all minimal idempotents of $E$ are conjugate (8.3.3). The main result here (8.3.4) asserts that the set of isomorphism classes of such $E$, with fixed unit group $i: G \rightarrow E$, is in a natural way the set of rational points of the Weyl chamber of $X\left(T_{0}\right) \otimes \mathbf{R}, T_{0}=(G, G) \cap T$ (relative to a fixed base $\Delta$ ).

Our final application of (6.5) is a numerical proof that any smooth, semisimple, algebraic monoid $E$ is isomorphic to $\operatorname{End}_{k}\left(k^{r}\right)$ for some $r>0$. 
2. Background. This section is a summary of relevant background information about algebraic varieties, algebraic groups and algebraic monoids.

(2.1) Algebraic varieties. $X, V, Z$ etc. will denote affine varieties over the algebraically closed field $k$ and $k[X]$ will denote the ring of regular functions on $X . X$ is irreducible if $k[X]$ is an integral domain and normal if further, $k[X]$ is integrally closed in its field of quotients. We shall assume $X$ is irreducible unless otherwise stated. A morphism $\varphi: X \rightarrow Y$ is dominant if $\varphi(X) \subseteq Y$ is dense and finite if $\varphi^{*}$ : $k[Y] \rightarrow k[X]$ is a finite extension of rings [12; §4.2]. Given $X$, there exists a unique morphism $\eta: \tilde{X} \rightarrow X$ such that $\eta$ is finite and birational and $\tilde{X}$ is normal.

Zariski's main theorem (referred to as ZMT) asserts that if $\varphi: U \rightarrow Y$ is finite-toone and birational and $Y$ is normal, then $\varphi$ is an open embedding.

If $X$ is normal the codim 2 condition [10, $\$ 5$, Lemma 1] asserts the following: If $U \subseteq X$ is open and $\operatorname{codim}_{X}(X \backslash U) \geqslant 2$, then any morphism $\varphi: U \rightarrow Y$, where $Y$ is affine, can be extended over $X$. ZMT and the codim 2 condition can sometimes be used together, to construct an isomorphism between the affine varieties $X$ and $Y$ (see (6.2) and (6.3)).

Let $\varphi: X \rightarrow Y$ be a morphism of varieties with $k^{*}$-action $(\varphi(t x)=t \varphi(x))$. Assume that the coordinate ring $A$ of $X$ (also $Y$ ) becomes graded in such a way that $A=\oplus_{n \geqslant 0} A_{n}$ and $A_{0}=k$. Let $0 \in Y$ be the unique fixed point. If $\varphi^{-1}(0)=\{p\}$ is a singleton, then $\varphi$ is a finite morphism. Since this result is closely related to Nakayama's lemma (it is the graded version) we refer to it as NAK.

(2.2) Algebraic groups. The basic reference for this section is [12]. We are especially concerned with reductive groups [12, Chapter 10].

An algebraic group is an affine, algebraic variety $G$ together with morphisms $m$ : $G \times G \rightarrow G$ and $i: G \rightarrow G$ such that $G$ becomes a group with $x y=m(x, y)$ and $x^{-1}=i(x)$. We shall always assume that $G$ is connected. $G$ is unipotent if it is isomorphic to a closed subgroup of $U(n)$, the set of matrices $\left(a_{i i}\right)$ of $\mathrm{Gl}_{n}(k)$ with $a_{i j}=0$ if $i>j$ and $a_{i i}=1$. Every algebraic group $G$ contains a maximal, normal, unipotent subgroup $R_{u}(G)[12, \S 19.5]$. $G$ is reductive if $R_{u}(G)=\{e\}$. An algebraic group $G$ is called a torus if $T \cong k^{*} \times \cdots \times k^{*}$; equivalently, $T$ is connected and consists entirely of semisimple (i.e. diagonalizable) elements [12, §15.3]. A maximal torus $T \subseteq G$ is a torus which is contained properly in no other. Any two maximal tori $S$ and $T$ of $G$ are conjugate [12, Corollary 21.3]. A Borel subgroup of $G$ is a maximal, connected, solvable subgroup $B$ of $G$. Any two Borel subgroups are conjugate [12, Theorem 21.3] and $G / B$ is a projective variety.

We now assume, unless otherwise stated, that $G$ is reductive. $G$ acts on itself by inner automorphisms, ad: $G \times G \rightarrow G,(g, h) \rightarrow g h g^{-1}$. Letting $g$ be the Lie algebra of $G$ [12, Chapter 3] we obtain the adjoint representation, Ad: $G \rightarrow \mathrm{Gl}(\mathrm{g})$, which is induced from ad. Ad $\left.\right|_{T}$ determines a direct sum decomposition

$$
\mathfrak{g}=\mathfrak{t} \oplus \coprod_{\alpha \in \Phi} \mathfrak{g}_{\alpha} \text {. }
$$

$\Phi \subseteq X(T)$ is the set of roots [12, p. 160] and $\operatorname{dim} \mathfrak{g}_{\alpha}=1$ for all $\alpha \in \Phi$. Furthermore, there exists a unique, one-dimensional unipotent subgroup $U_{\alpha} \subseteq G$ such that $\mathfrak{g}_{\alpha}=$ $\mathscr{Z}_{1}\left(U_{\alpha}\right)$ (tangent space at the identity). Choosing a Borel subgroup $B$ of $G$ with 
$T \subseteq B$ is the same as choosing a base $\Delta$ of $\Phi[12, \S 27.3]$. Every $\lambda \in \Phi$ has a unique expression of the form $\lambda=\sum_{\alpha \in \Delta} c_{\alpha} \alpha$, where $\left\{c_{\alpha}\right\} \subseteq \mathbf{Z}^{+}$or $\mathbf{Z}^{-} . \Delta$ thus determines a partition $\Phi=\Phi^{+} \cup \Phi^{-}$into positive and negative roots. Here, $\Phi^{+}=\left\{\lambda \in \Phi \mid c_{\alpha} \geqslant 0\right.$ for all $\alpha \in \Delta\}$ and $\Phi^{-}=-\Phi^{+} . B$ is then the subgroup generated by $T$ and $\left\{U_{\alpha} \mid \alpha \in\right.$ $\left.\Phi^{+}\right\} . B^{-}$, the opposite Borel subgroup, is generated by $T$ and $\left\{U_{\alpha} \mid \alpha \in \Phi^{-}\right\}[12, \S 26.2]$.

$W=N_{G}(T) / T$ is the Weyl group and is generated by the set of reflections $\left\{\sigma_{\alpha} \mid \alpha \in \Delta\right\}$ for any choice of $\Delta$ [12, Appendix A5].

A representation $(\rho, V)$ of $G$ is a morphism $\rho: G \rightarrow \operatorname{Gl}(V) .(\rho, V)$ (or just $\rho$ ) is irreducible if $V$ contains no proper, nonzero, $G$-stable subspace. If $G$ is semisimple there is a one-to-one correspondence

irreducible representations of $G \leftrightarrow$ fundamental weights $X^{+}(T)$.

Here $X^{+}(T)=\left\{\left.\lambda \in X(T)\right|_{(\lambda, \alpha) \geqslant 0}\right.$ for all $\left.\alpha \in \Delta\right\}$ and (, ) is a $W$-invariant quadratic form on $X(T)$. Notice the dependence on the choice of $T$ and $B=B(\Delta)$. The correspondence is as follows: For each irreducible representation $(\rho, V)$ there exists $\lambda \in X^{+}(T)$ and a line $L \subseteq V$ such that $\rho(g)(v)=\lambda(g) v$ for all $g \in B$ and all $v \in L$ $[12,31.4]$. Here $v \neq 0$ is called a highest weight vector with highest weight $\lambda$. The terminology is justified because $\lambda-\mu$ is a sum of positive roots for all other weights of $\left.\rho\right|_{T}[12]$.

(2.3) Algebraic monoids. The interest in algebraic monoids (other than toric monoids $[5,14,18]$ and convenient encounters $[7,32])$ is quite recent. The reader will be referred to the recent work of Putcha and myself for the proofs of many assertions.

An algebraic monoid $E$ is an affine, algebraic variety together with an associative morphism $m: E \times E \rightarrow E$ and a two-sided unit $1 \in E$ for $m$.

$G=G(E)=\left\{x \in E \mid x^{-1} \in E\right\}$ is an open algebraic subgroup of $E$ and there exists a morphism $\chi: E \rightarrow k$ such that $\chi^{-1}\left(k^{*}\right)=G[19, \mathrm{I}$, Theorem 1.1]. $I(E)=\{e$ $\left.\in E \mid e^{2}=e\right\}$ is the set of indempotents of $E$. If $x \in E$ and $H \subseteq G$ is a subgroup, then $\mathrm{Cl}_{H}(x) \subseteq E$ is the $H$-conjugacy class of $x$ in $E$. A $D$-monoid $Z$ is an irreducible, algebraic monoid such that $G(Z)=T$ is a torus (2.2). The normal $D$-monoids are precisely the affine torus embeddings $[14,18], X(Z)=\operatorname{Hom}(Z, k)$, as algebraic monoids, is the set of characters of $Z . Z$ is determined to within an isomorphism by the commutative monoid $X(Z)[20, \S 2]$ and $k[Z]$ is the monoid algebra of $X(Z)$ over $k$. It turns out that $Z$ is a normal variety if and only if for $\chi \in X(T)$ and $\chi^{n} \in X(Z)$ we have $\chi \in X(Z)[14$, Chapter 1 , Lemma 1]. We say that $X(Z)$ is normal in this case. If $E$ is any algebraic monoid, a maximal $D$-submonoid $Z \subseteq E$ is a $D$-monoid that is contained properly in no other. Equivalently, $Z=\bar{T} \subseteq E$, where $T \subseteq G$ is a maximal torus. This makes it clear that all maximal $D$-submonoids are conjugate [12, Corollary 21.3].

If $Z$ is a two-dimensional $D$-monoid with $0 \in Z$, then $I(Z)=\{1, e, f, 0\}$ with $e f=0[19$, II, Theorem 3.20]. If $E$ is any irreducible, algebraic monoid then by [20, Theorem 1.8]

$$
I(E)=\bigcup_{T}\{I(\bar{T}) \mid T \subseteq G \text { a maximal torus }\} .
$$


A monoid $E$ is regular if for all $x \in E$ there exists $g \in G=G(E)$ and $e \in I(E)$ such that $g e=x$. This is not the usual definition, but is equivalent to it for irreducible monoids [21, Theorem 13]. An irreducible, algebraic monoid $E$ is reductive if $G(E)$ is a reductive group. From the results of [23 and 29] it follows that any reductive monoid is regular. This should be regarded as the most fundamental general fact in the theory. As an immediate corollary it follows that $E=\cup_{e \in I(\bar{T})} G e G$ for any reductive monoid $E$. In particular, $E$ has no more nonzero nilpotent ideals.

(2.3.1) Definition. An algebraic monoid $E$ is semisimple if $G(E)$ is reductive, $\operatorname{dim} Z G(E)=1, E$ is irreducible, $0 \in E$ and $E$ is normal.

Comment. The two important restrictions here are reductivity and irreducibility. Nonnormal monoids have a lot in common with their normalization. The restriction on the center is perhaps somewhat artificial but in general $\overline{Z G(E)^{0}}$ is a $D$-monoid and can best be studied with the techniques of [14]. Any reductive monoid $E$ is isogenous to a product $E^{\prime} \times H$, where $H$ is a reductive group and $E^{\prime}$ is a reductive monoid with 0 ; so having a 0 is mainly for convenience.

(2.3.2) [30, Corollary 2.2] Let $E$ be a two-dimensional, noncommutative algebraic monoid without 0 . Then there are two mutually exclusive possibilities for $e \in$ $I(E) \backslash\{1\}$ :

(a) $E e=\{e\}$ and $e E=\mathrm{Cl}(e)$ or

(b) $e E=\{e\}$ and $E e=\mathrm{Cl}(e)$.

In both cases $E=G \cup \mathrm{Cl}(e)$.

This occurs quite often as follows: Let $e \in \bar{T} \subseteq \bar{B}$ and $U_{\alpha} \subseteq B, \alpha \in \Phi^{+}$, and suppose $e U_{\alpha} \neq U_{\alpha} e$. Choose $k^{*} \subseteq T$ such that $e \in \bar{k}^{*}$ [14, Chapter 1, Theorem 2]. Then $E=\overline{k^{*} \cdot U_{\alpha}} \subseteq \bar{B}$ satisfies the assumptions of (2.3.2). The example to keep in mind is the set of $2 \times 2$ upper triangular matrices $\left(a_{i j}\right)$ and $a_{11}=1$.

(2.3.2) is one starting point in our construction of the "big cell".

3. Polyhedral root systems. This section is technical. The necessary results from torus embeddings and representation theory are synthesized to yield some tools needed in the proofs of our main results. We establish an important relationship between the set of weights $\Phi(\rho)$ of an irreducible representation $\rho$ of $G$ and the convexity properties of $X \overline{(\rho(T))}$. It turns out that minimal idempotents of $\overline{\rho(T)}$ correspond to highest weight vectors of $\rho$.

We also define the fundamental notion of a polyhedral root system (3.6).

Definition. [14]. An affine, torus embedding $Z$ is a normal, algebraic variety that contains a dense open torus $i: T \rightarrow Z$ such that the action $T \times T \rightarrow T,(x, y) \rightarrow x y$, extends to a morphism $T \times Z \rightarrow Z$.

It is a simple fact [18, Proposition 3.3] that an affine torus embedding $Z$ is a normal $D$-monoid, but conversely, any normal $D$-monoid is an affine torus embedding. The elementary results of [14 and 18] will thus be used freely in our discussion of $D$-monoids.

(3.1) Lemma. Let $Z$ be a normal D-monoid with 0 . Then there exists $\left\{\chi_{i}\right\}_{i=1}^{s} \subseteq X(Z)$ such that

(i) for all $\chi \in X(Z), \chi^{m}=\prod_{i=1}^{s} \chi_{i}^{m_{i}}$ for some $m$ and $m_{i} \geqslant 0$, 
(ii) $\chi_{i} \in I \backslash I^{2}$ for all $i$, where $I=\{f \in k[Z] \mid f(0)=0\}$,

(iii) $s$ is minimal with properties (i), (ii). Furthermore, $\left\{\chi_{i}\right\}$ is unique.

The proof is written down in [27] but follows also from [18, §1.3].

$\left\{\chi_{i}\right\}$ is the set of fundamental generators. The reader is encouraged to consider the following geometric interpretation.

Given $Z$ as in the lemma, there is a convex, rational, polyhedral cone $\sigma \subseteq X(Z) \otimes$ $Q$ such that $X(Z)=\sigma \cap X(T)$ [14, Chapter 1]. $\sigma$ is the rational, convex hull of a finite number of rays $R_{i} \subseteq \sigma, i=1, \ldots, s$, and for each $i$ there is a unique element $\chi_{i} \in R_{i} \cap X(Z)$ (the $\chi_{i}$ 's of (3.1) it turns out) with minimal distance from the origin. Here $Z \cong\left\{(a, b, c) \in k^{3} \mid a c=b^{3}\right\}$.

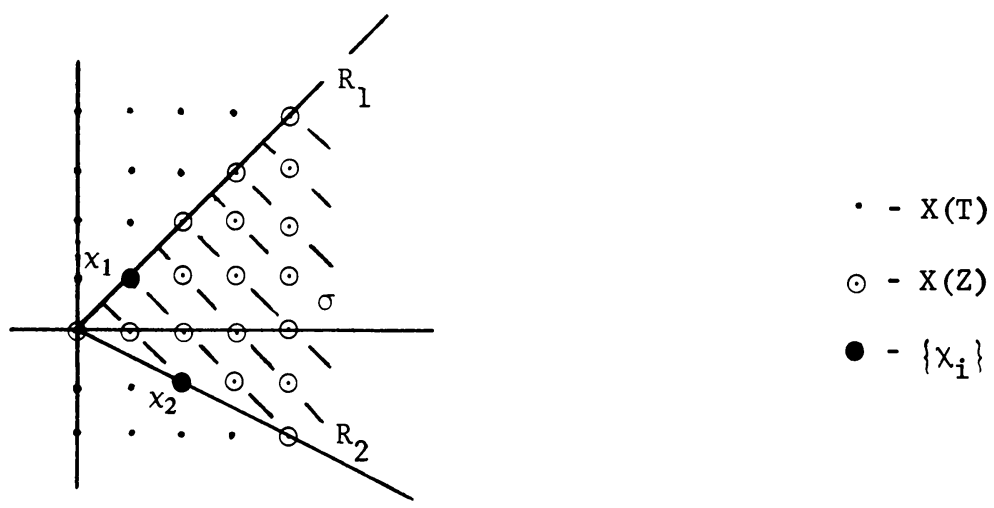

FIGURE 3

For the normal $D$-monoid $Z$, let $I_{1}(Z)=\{e \in I(Z) \mid e \neq 0$ and $e$ is minimal $\}$. For each $e \in I_{1}(Z), \operatorname{dim}(e Z)=1[19$, II, Lemma 3.11] and so $e \cdot Z \cong k$. Hence, we obtain a character $\chi_{e}: Z \rightarrow k, \chi_{e}(z)=e \cdot z$.

(3.2) Corollary. $\left\{\chi_{e} \mid e \in I_{1}(Z)\right\}$ is the set of fundamental generators.

This proof is also written down in [27]. In fact, there we prove directly that properties (i)-(iii) of (3.1) are satisfied for $\left\{\chi_{e}\right\}$. This canonical relationship between $I_{1}(Z)$ and the one-dimensional faces of $\sigma$ can be extended to yield a canonical bijection of lattices

$$
\mathscr{F}(\sigma) \leftrightarrow I(Z),
$$

where $\mathscr{F}(\sigma)$ denotes the set of faces of $\sigma$ (see [18, $\S 4$ and 14, Chapter 1] for more discussion).

Let $G$ be a reductive group and $T \subseteq G$ a maximal torus. If $\rho: G \rightarrow \mathrm{Gl}(V)$ is a representation we let $\Phi(\rho) \subseteq X(T)$ denote the set of weights of $\left.\rho\right|_{T}$. By definition, $V=\oplus_{\alpha \in \Phi(\rho)} V_{\alpha}$, where $(0) \neq V_{\alpha}=\{|v \in V| \rho(t)(v)=\alpha(t) v$ for all $t \in T\}$. $\langle\Phi(\rho)\rangle \subseteq X(T)$ denotes the submonoid of $X(T)$ generated by $\Phi(\rho)$.

(3.3) Lemma. $Z=\overline{\rho(T)} \subseteq \operatorname{End}(V)$ is a D-submonoid with $X(Z)=\langle\Phi(\rho)\rangle$.

For the proof see [30, Lemma 4.1]. 


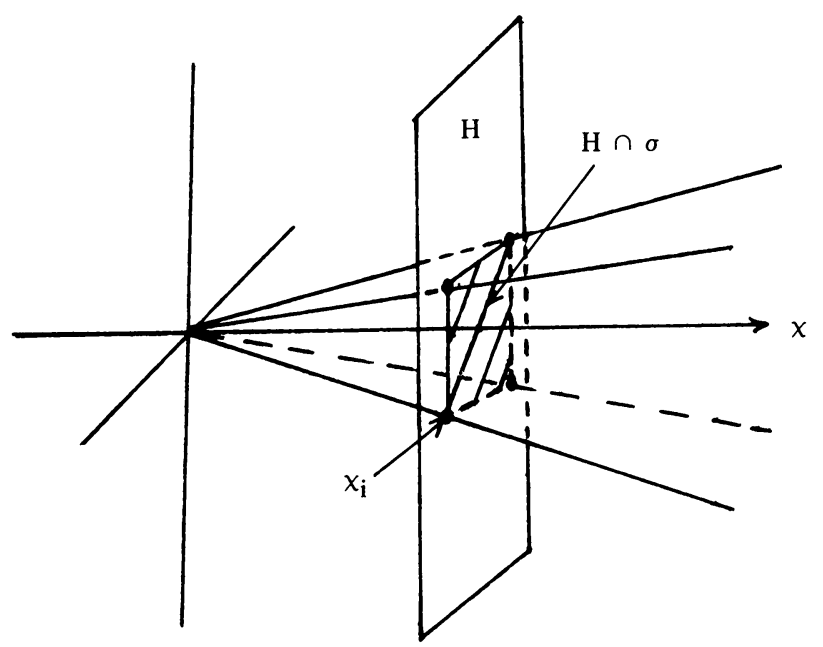

Figure 4

(3.4) Let $G_{0}$ be a semisimple group and $\rho^{\lambda}: G_{0} \rightarrow \mathrm{Gl}(V)$ an irreducible representation with highest weight $\lambda$ (with respect to $T \subseteq B=B(\Delta)(2.2)$ ). For $n>0$ define $\rho_{n}^{\lambda}: G \rightarrow \mathrm{Gl}(V)$ by

$$
\rho_{n}^{\lambda}(g, t)=\rho(g)\left[\begin{array}{lll}
t^{n} & & 0 \\
& \ddots & \\
0 & & t^{n}
\end{array}\right], \quad \text { where } G=G_{0} \times k^{*} .
$$

It is easy to check that $\Phi\left(\rho_{n}^{\lambda}\right)=\left\{(\alpha, n) \mid \alpha \in \Phi\left(\rho^{\lambda}\right)\right\} \subseteq X(T)=X\left(T_{0} \times k^{*}\right)=$ $X\left(T_{0}\right) \oplus \mathbf{Z}$, and thus, by (3.3),

$$
X \overline{\left(\rho_{n}^{\lambda}(T)\right)}=\left\langle\left\{(\alpha, n) \mid \alpha \in \Phi\left(\rho^{\lambda}\right)\right\}\right\rangle .
$$

Let $\lambda \in \Phi\left(\rho^{\lambda}\right)$ be the highest weight. By [25, Theorem 1(6)] and (3.2), $Z \rightarrow k=$ $\operatorname{End}(k \cdot v), t \rightarrow \rho(t): v \rightarrow \rho(t) v$ is a fundamental generator of $\overline{\rho_{n}^{\lambda}(T)}=Z$ if $v \in V$ is a maximal vector of $\lambda$.

(3.5) Proposition. $\Phi\left(\rho_{n}^{\lambda}\right) \subseteq$ rational convex hull of $W \cdot(\lambda, n)$, where $W$ is the Weyl group.

Proof. By [25] $W$ acts transitively on $I_{1}(Z)$ (since $Z$ "comes from" an irreducible representation of $G_{0},[25]$ applies). Thus by (3.2) $W$ acts transitively on $\left\{\chi_{i}\right\}$. But from our geometric description of $X(Z)$ and $\left\{\chi_{i}\right\}$ (following (3.1)) it follows that the rational convex hull of $\left\{\chi_{i}\right\}$ is the hyperplane section of $X(T) \otimes \mathbf{Q} \cap \sigma$ of distance $n$ from the $X\left(T_{0}\right)$-hyperplane, orthogonal to the $x$-axis (see Figure 4). Thus, the result follows from (3.3), since every element of $\Phi\left(\rho_{n}^{\lambda}\right)$ has $x$-coordinate $n$.

Alternate PRoOf (SINCE [25] MAY NOT APPEAR). Assume there exists $\mu \in \Phi\left(\rho^{\lambda}\right)$ with $\Phi\left(\rho^{\lambda}\right) \subseteq$ rational convex hull of $W \cdot \mu$ (and that can be deduced from [11, Lemma $13.2 \mathrm{~A}$ ] in all characteristics).

It suffices to prove that $\mu=\lambda$, since the result is translation invariant and $\Phi\left(\rho_{n}^{\lambda}\right)=\Phi\left(\rho^{\lambda}\right)+(0, n) \subseteq X\left(T_{0}\right) \oplus \mathbf{Z}$. Call $\mu$ the extreme weight. 
Suppose $\lambda \neq \mu$. Then $\lambda=\mu+p$, where $p=\sum_{\alpha \in \Phi^{+}} m_{\alpha} \alpha$, and $\Phi^{+}$is the set of positive roots [12, Appendix A10] and $\left\{m_{i}\right\} \subseteq \mathbf{Q}^{+}$with $\sum m_{i}>0$ (since $\lambda$ is the highest weight).

On the other hand, $\lambda=\sum_{\tau \in W} r_{\tau} \tau(\mu)$, where $\sum_{\tau \in W} r_{\tau}=1$ and $r_{\tau} \geqslant 0$ (since $\mu$ is the extreme weight).

But then for all $\tau \in W$

$$
r_{\tau}(\mu+p)>r_{\tau}(\mu) \geqslant r_{\tau}(\tau(\mu))
$$

by Appendix A.10 of [12]. Thus,

$$
\lambda=\mu+p=\sum_{\tau \in W} r_{\tau}(\mu+p)>\sum_{\tau \in W} r_{\tau} \tau(\mu)=\lambda .
$$

This contradiction concludes the proof.

Definition. A root system of rank $n$ is an ordered pair $(X, \Phi)$, where

(i) $X$ is a finitely generated free abelian group of rank $n$.

(ii) $\Phi \subseteq X$ is finite, spans $X$ over $\mathbf{Q}$ and does not contain 0 .

(iii) For all $\alpha \in \Phi$ there exists an involution $\sigma_{\alpha}: X \rightarrow X$ such that $\sigma_{\alpha}(\alpha)=-\alpha$ and rank $X^{\sigma_{\alpha}}=\operatorname{rank} X-1$.

(iv) The only rational multiples in $\Phi$ of $\alpha \in \Phi$ are $\pm \alpha$.

(v) For all $\alpha \in \Phi$ and $x \in X, \sigma_{\alpha}(x)-x$ is an integral multiple of $\sigma$.

REMARK. This is not the customary definition (see [12, Appendix]). What we have here is an axiomatic characterization for $(X(T), \Phi(T))$ of a semisimple group, and thus, by [12, Theorem 32] the precise classification of such groups. This tidbit of heresy is exactly what is needed to resolve the ambiguity of the fundamental group $[12, \S 31.1]$.

(3.6) Main Definition. A polyhedral root system of semisimple rank $n$ is a triple $(X, \Phi, C)$, where:

(i) $X$ is a free abelian group of rank $n+1$.

(ii) $\Phi \subseteq X$ spans a subgroup ( $\Phi)$ of $X$ of rank $n$.

(iii) $C \subseteq X$ is the intersection with $X$ of a rational polyhedral cone $\sigma$ of dimension $n+1$ of $X \otimes Q$.

(iv) $\left(X_{0}, \Phi\right)$ is a root system in the above sense, where $X_{0}=\{\chi \in X \mid m \chi \in(\Phi)$ for some $m \in \mathbf{Z}\}$.

(v) For each $\sigma \in \Phi$, the reflections $\sigma_{\alpha}: X_{0} \rightarrow X_{0}$ can be extended to $X$ so that $\sigma_{\alpha}(C)=C$.

$C$ as in (iii) will be called a polyhedral cone (integrality being understood).

Comments. (1) A polyhedral root system $(X, \Phi, C)$ determines a torus embedding $T=\operatorname{Spec}(k[X]) \rightarrow Z=\operatorname{Spec}(k[C])$ with $W$-action, where $W$ is the finite group generated by $\left\{\sigma_{\alpha} \mid \alpha \in \Phi\right\}$. Here, $k[\cdots]$ denotes "monoid algebra".

(2) By [12, Appendix A1], each $\sigma_{\alpha}$ is unique since $\sigma_{\alpha}(\Phi \cup\{\chi\})=\Phi \cup\{\chi\}$, and $\Phi \cup\{\chi\}$ spans $X$ over $Q$. Here, $\chi=\Sigma \chi_{i} \in X$, where $\left\{\chi_{i}\right\}$ is the set of fundamental generators (3.1).

(3) Polyhedral root systems are quite abundant: Let $\left(X_{0}, \Phi\right)$ be a root system and choose a finite subset

$$
S \subseteq X_{0} \oplus \mathbf{N} \subseteq X_{0} \oplus \mathbf{Z}=X
$$


Let $S^{\prime}=\{(w \cdot \alpha, n) \mid w \in W,(\alpha, n) \in S\}$ and let $C_{S} \subseteq X$ be the smallest polyhedral cone containing $S^{\prime}$. It is an easy exercise to check that $\left(X, \Phi, C_{S}\right)$ is a polyhedral root system.

(4) $(X, \Phi, C)$ is a polyhedral root system of type $G$ if $(X, \Phi)$ is isomorphic to $(X(T), \Phi(T)$ ) for some (any) maximal torus $T \subseteq G$. By definition we are considering only the polyhedral root systems of type $G$ for $G$ reductive with $\operatorname{dim} Z G=1$.

(3.7) Proposition. Let E be a semisimple, algebraic monoid (2.3.1) with maximal torus $T$ and maximal $D$-submonoid $Z=\bar{T} \subseteq E$. Assume that $Z$ is normal. Then $(X(T), \Phi(T), X(Z))$ is a polyhedral root system of type $G=G(E)$.

Proof. Let $W=N_{G}(T) / T$. Then $W$ acts on $T$ and thus on $Z$, by continuity. Let us verify the axioms of (3.6) for $(X(T), \Phi(T), X(Z))$. (i) is obvious. (ii) follows from the facts that $\operatorname{rank} X_{0}=\operatorname{rank} X-\operatorname{dim} Z G$ and $\operatorname{dim} Z G=1$. (iii) follows since $Z$ is a torus embedding with 0 (note that if $0 \notin Z, X(Z)$ is not a cone). To prove (iv) we observe that $\left(X_{0}, \Phi\right)$ (of $(X(T), \Phi(T), X(Z))$ ) is the root system of $G /(Z G)^{0}$. Finally, for (v), we notice that $\sigma_{\alpha}(X(Z))=X(Z)$ for all $\alpha \in \Phi$ by our initial remark.

Remark. It will be proved in (6.4) that $Z$ is always normal for a semisimple monoid $E$. This is a little delicate since there are no general results from algebraic geometry that assert " $V$ normal $\Rightarrow V^{T}$ normal" for affine varieties $V$ with torus action, $T \times V \rightarrow V$. That $Z=\left(E^{T}\right)_{\text {red }}$ is normal is perhaps even more surprising since in general, $E^{T}$ is not reduced as a scheme.
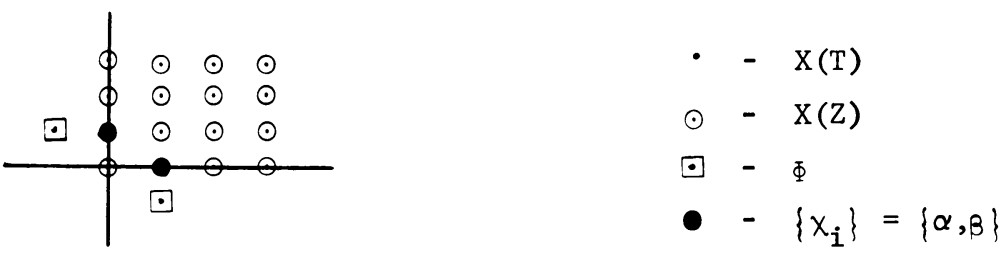

FigURE 5

(3.8) Examples. (1) Let $E=\operatorname{End}_{k}\left(k^{2}\right)$, viewed as a semisimple, algebraic monoid. Then $T=\left\{\left[\begin{array}{cc}a & 0 \\ 0 & b\end{array}\right] \mid a b \neq 0\right\}, Z=\left\{\left[\begin{array}{cc}a & 0 \\ 0 & b\end{array}\right]\right\}, X(T)=\mathbf{Z} \oplus \mathbf{Z}$, generated by $\alpha:\left[\begin{array}{ll}a & 0 \\ 0 & b\end{array}\right] \rightarrow a$ and $\beta:\left[\begin{array}{ll}a & 0 \\ 0 & b\end{array}\right] \rightarrow b, \Phi=\{(1,-1)=\alpha-\beta,(-1,1)=\beta-\alpha\}$ and $X(Z)=\mathbf{N} \alpha \oplus \mathbf{N} \beta \subseteq$ $\mathbf{Z} \oplus \mathbf{Z}$. Geometrically, we have Figure 5 .

(2) Similarly, one can compute the polyhedral root system $(X, \Phi, C)$ of $E=$ $\operatorname{End}_{k}\left(k^{r}\right)$. Let $\left\{e_{i}\right\}_{i=1}^{r}$ be a basis of $\mathbf{Z}^{r}$. Then

$$
(X, \Phi, C) \cong\left(\mathbf{Z}^{r},\left\{e_{i}-e_{j}\right\}_{i \neq j},\left\langle e_{i}\right\rangle\right) .
$$

(3) Let $G_{0}$ be the adjoint, simple group of type $B_{2}$ and let $\rho=\mathrm{Ad}: G_{0} \rightarrow \mathrm{Gl}(\mathrm{g})$ be the adjoint representation. Define

$$
E=\text { normalization of } \overline{\rho\left(G_{0}\right) \cdot k^{*}} \subseteq \operatorname{End}(g),
$$

where $k^{*} \subseteq \operatorname{End}(\mathrm{g})$ denotes the invertible scalar transformations. Then $G(E) \cong G_{0}$ $\times k^{*}$ since $G_{0}$ is of adjoint type. It is easily checked using (2.2) and Lemma (3.3), 
that

$$
X(Z)=\langle(\alpha, 1)\rangle_{\alpha \in \Phi} \subseteq X(T)=X\left(T_{0}\right) \oplus \mathbf{Z}
$$

where $T \subseteq G(E)$ is a maximal torus and $T_{0}=T \cap G_{0}$. In this case, the picture is as in Figure 6.

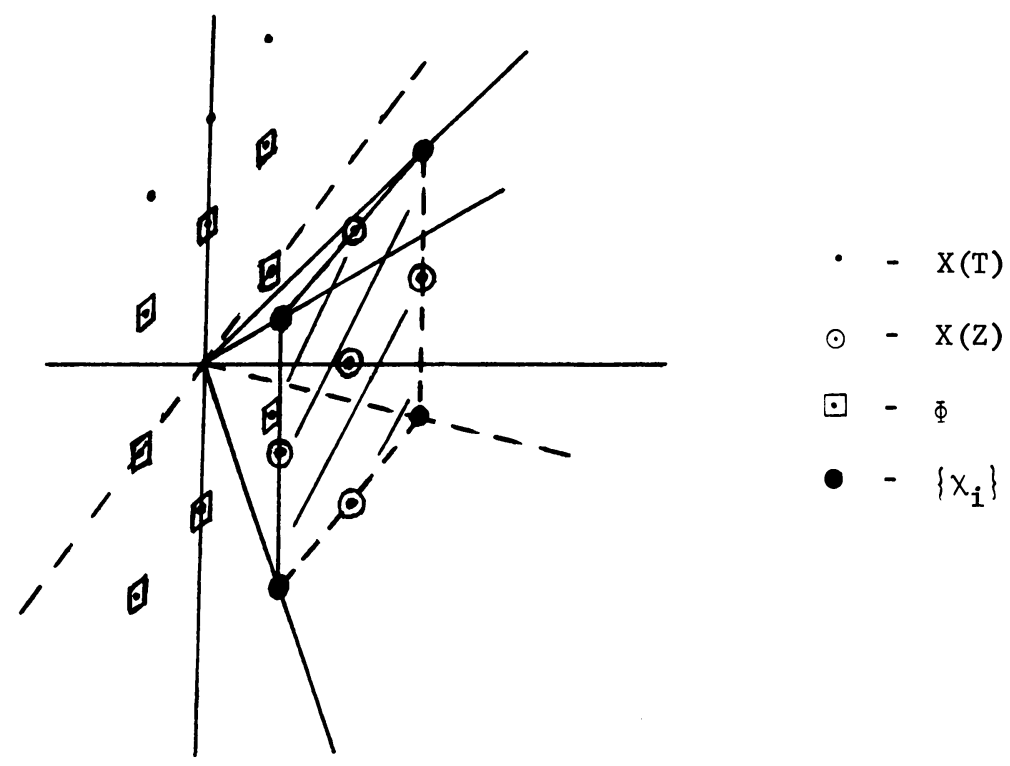

FIGURE 6

4. Big cell. The actual construction of all semisimple monoids will be accomplished by applying the results of $\S 3$. This will leave us with the problem of proving that different monoids have different polyhedral root systems.

In this section we establish a procedure for constructing morphisms from a given normal, reductive, algebraic monoid (see (4.5)). The constructive element in the proof is inspired by Chevalley's notion of a "big cell” [12, §28.5]. My big cell contains Chevalley's as an open subset, and is big enough so that we may apply the codim 2 condition of (2.1) to the problem of extending certain morphisms.

We begin the section with a summary of some important ideas of M. S. Putcha [24] relating the Borel subgroups $\{B \mid B \supseteq T\}$ to the idempotents of $\bar{T}$.

$E$ will always be a reductive monoid and $B \subseteq G=G(E)$ a Borel subgroup with maximal torus $T \subseteq B$. Let $Z=\bar{T} \subseteq E$. Define

$$
\Lambda=\Lambda(B)=\{e \in I(Z) \mid g e=\text { ege for all } g \in B\}
$$

Then

$$
B=\{g \in G \mid g e=e g e \text { for all } e \in \Lambda\}
$$


and $\Lambda \cap \mathrm{Cl}_{W}(e)$ contains exactly one element for each $e \in I(Z)$. (See [26, Theorem 1.2]). The result can also be deduced from the results of [24].) $\Lambda=\Lambda(B)$ is called a cross-section lattice associated with $B$. This terminology is well chosen because by $[\mathbf{2 4}$, Theorem 11], any subset $S \subseteq I(Z)$ with the properties

(i) $S \rightarrow I(Z) \rightarrow I(Z) / W$ is bijective,

(ii) if $e, f \in S$ and $e \sigma f \sigma^{-1}=\sigma f \sigma^{-1}$ for some $\sigma \in W$, then ef $=f$,

is conjugate to $\Lambda ; S=w \Lambda w^{-1}$ by some unique element $w \in W$. Here, $I(Z) / W$ denotes the set of conjugacy classes of $I(Z)$ under the action of $W$ by inner automorphisms.

Cross-section lattices are the algebraic monoid way of looking at the set of Borel subgroups that contain a given maximal torus. It is my opinion that the cross-section lattice will be one of the most important finite invariants relating the monoid to the geometry of the root system.

(4.1) Lemma. Let $e \in I(Z)$ and $\sigma_{\alpha} \in \Phi(T)$. Then there exists an irreducible, closed, algebraic submonoid $E_{e, \alpha} \subseteq E$ such that

(a) $U_{\alpha}, U_{-\alpha} \subseteq E_{e, \alpha}$,

(b) $e \in E_{e, \alpha}$,

(c) $\operatorname{dim} E_{e, \alpha}=4$.

ProOF. We start with some simple properties of the automorphism $\sigma_{\alpha}: T \rightarrow T$.

(i) There exists a unique, closed, irreducible one dimensional subgroup $H \subseteq T$ such that $\sigma_{\alpha}(t)=t^{-1}$ for all $t \in H$ (since $\sigma_{\alpha}$ is a reflection).

(ii) If $k^{*} \subseteq T$ is any one-parameter subgroup, then $K=k^{*} \cdot \sigma_{\alpha}\left(k^{*}\right)$ is a closed, $\sigma_{\alpha}$-stable subgroup of dimension $\leqslant 2$.

Now let $k^{*} \subseteq T$ be any one-parameter subgroup such that the dotted arrow exists with $\lambda(0)=e$;

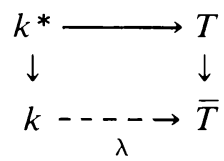

Such a $k^{*}$ exists by [14, Chapter 1 , Theorem 2]. Let $K=k^{*} \sigma_{\alpha}\left(k^{*}\right)$ as in (ii).

Case $1 . \operatorname{dim} K=1$. In this case we must have $\sigma_{\alpha}(t)=t$ for all $t \in K$ since otherwise $\sigma_{\alpha}(t)=t^{-1}$. But then we would have morphisms

$$
\underset{\lambda}{\rightarrow \rightarrow} \bar{T}, \quad k \underset{\sigma_{a} \circ \lambda}{\rightarrow} \bar{T}
$$

such that $\sigma_{\alpha} \circ \lambda(t)=\lambda\left(t^{-1}\right)$ for all $t \in k^{*}$ which together yield a morphism $\mathbf{P}^{1} \rightarrow \bar{T}$, which is absurd since $\bar{T}$ is affine (see [12, §21.2]).

So let $E_{e, \alpha}=k^{*} \cdot G_{\alpha}$, where $G_{\alpha}=\left\langle U_{\alpha}, U_{-\alpha}\right\rangle$ is the subgroup generated by $U_{\alpha}$ and $U_{-\alpha}$. By [12, Corollary 32.2], $G_{\alpha} \cong \mathrm{Sl}_{2}(k)$ or $\mathrm{PGl}_{2}(k)$, and since $k^{*}$ normalizes $U_{\alpha}$ and $U_{-\alpha}, k^{*} \cdot G_{\alpha} \subseteq G$ is a subgroup. $\operatorname{dim} E_{e, \alpha}=4$ since otherwise $G\left(E_{e, \alpha}\right)=G_{\alpha}$, and by [35] $e \notin \overline{G_{\alpha}}$. But $e \in \overline{k^{*}} \subseteq E_{e, \alpha}$. 
Case 2. $\operatorname{dim} K=2$. In this case $\sigma_{\alpha}: K \rightarrow K$ is a reflection. Thus, there exist $\sigma_{\alpha}$-stable one-parameter subgroups $H, M \subseteq K$ such that $H \cdot M=K, \sigma_{\alpha}(t)=t^{-1}$ for $t \in H$, and $\sigma_{\alpha}(t)=t$ for $t \in M$. But $G_{\alpha}$ (as in Case 1) contains such a subgroup as $H$ and by (i) it must be $H ; H \subseteq G_{\alpha}$. In this case we let $E=\overline{M \cdot G_{\alpha}} \subseteq E$. It follows easily, since $e \in \overline{M \cdot H} \subseteq E_{e, \alpha}$, that (a)-(c) are satisfied.

(4.2) Lemma. Suppose $e \in I(Z)$ is a maximal idempotent $(e \neq 1)$ and $\alpha \in \Phi$. Then either $e U_{\alpha} \neq\{e\}$ or $U_{\alpha} e \neq\{e\}$.

Proof. Let $E_{e, \alpha} \subseteq E$ be as in (4.1). Then $G\left(E_{e, \alpha}\right)$ is isogenous to $\mathrm{Gl}_{2}(k)$ since it is nonabelian, reductive and has dimension 4 [12, §32, Example 5].

Case 1. $E_{e, \alpha}$ has a zero element (of its own). By [19, II, Theorem 3.20], if $M \subseteq G\left(E_{e, \alpha}\right)$ is a maximal torus with $e \in \bar{M}$, then $I(\bar{M})=\left\{1, e_{1}, e_{2}, 0\right\}$, where 0 is the zero element of $E_{e . \alpha}$. Since $e$ is maximal, we must have $e=e_{1}$ or $e_{2}$. But from (2.3.2) either $e U_{\alpha} \neq\{e\}$ or $U_{\alpha} e \neq\{e\}$.

Case 2. $E_{e, \alpha}$ does not have a zero element. Then from [30, Theorem 5.5] or the proof of [28, Theorem 3.2] we must have $I\left(E_{e, \alpha}\right)=\{1, e\}$ and

$$
G_{\alpha} \times \bar{S} \rightarrow E_{e, \alpha}, \quad(g, s) \rightarrow g \cdot s,
$$

is a finite, dominant morphism, where $S$ is the identity component of the center of $G\left(E_{e, \alpha}\right)$. Since $e \in \bar{S}$ we see that both $e U_{\alpha} \neq\{e\}$ and $U_{\alpha} e \neq\{e\}$ in this case.

(4.3) Corollary. Let $e, \alpha$ and $Z$ be as in (4.2) and suppose $e \in \Lambda=\Lambda(B)$, the cross-section lattice associated with $B$. Then

$$
B_{u} \rightarrow e B_{u}, \quad u \rightarrow e u,
$$

is bijective, where $B_{u} \subseteq B$ is the unipotent radial of $B$.

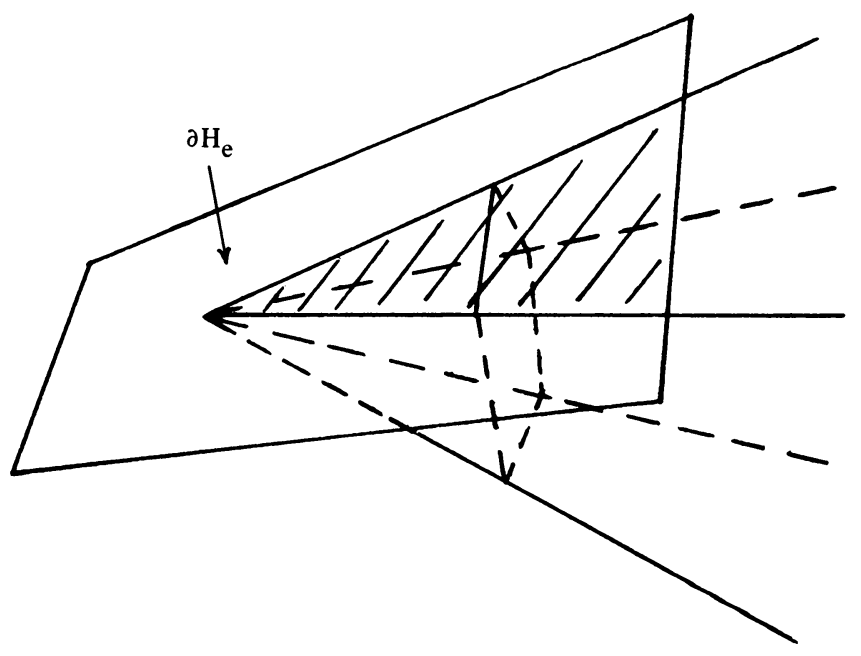

FIGURE 7 
Proof. Let $U=\left\{u \in B_{u} \mid e u=e\right\}$. Then $U \subseteq B_{u}$ is a closed subgroup. If $t \in T$ and $u \in U$, then $e t u t^{-1}=$ teut $^{-1}=$ tet $^{-1}=e$; so $T$ normalizes $U$. Thus, by [12, Proposition 28.1] $U=\prod_{\alpha \in S} U_{\alpha}$, where $S=\left\{\alpha \in \Phi^{+} \mid e U_{\alpha}=\{e\}\right\}$.

Let $\alpha \in S$. By the definition of $\Lambda(B)$ we must have eue $=u e$ for all $u \in U_{\alpha}$. Hence, $U_{\alpha} e=\{e\}$. But, this contradicts (4.2). Thus, $S$ is empty.

Definition-Lemma. For $e \in I(Z)$ a maximal idempotent $(e \neq 1)$, there exists a unique open submonoid $Z_{e} \subseteq Z$ such that $I\left(Z_{e}\right)=\{1, e\}$. In fact, $Z_{e}=T \cup T e$.

Proof. $T \cup T e$ is a submonoid and it is open since $Z \backslash(T \cup T e)=\bigcup_{f \in I(Z) \backslash\{1, e\}} Z f$ is closed (see [18, §4] for more details).

Geometrically, $Z_{e} \subseteq Z$ represents one of the canonical half-spaces $H_{e}$ that contain the rational, polyhedral cone $\sigma$ of $X(Z)$, and whose boundary intersects it in a subset of codimension one.

We can now prove the main technical result of this section.

(4.4) TheOrem (BIG Cell). Suppose $E$ is reductive and normal, and $e \in I(Z) \cap$ $\Lambda(B)$ is a maximal idempotent $(Z=\bar{T}$ and $T \subseteq B)$. Let $B^{-}$be the opposite Borel subgroup. Define $m: B_{u}^{-} \times Z_{e} \times B_{u} \rightarrow E$ by $m(x, y, z)=x y z$. Then $m$ is an open embedding.

Proof. We note, first of all, that

$$
B_{u}^{-} \rightarrow B_{u}^{-} e, \quad u \rightarrow u e,
$$

is bijective. The proof is the same as (4.3) since $B^{-}=\{g \in G \mid e g=e g e$ for all $e \in \Lambda$ \} (see [22, Theorem 4.5]).

Suppose then that $u_{1} x_{1} v_{1}=u_{2} x_{2} v_{2}$, where $u_{1}, u_{2} \in B_{u}^{-}, v_{1}, v_{2} \in B_{u}$ and $x_{1}, x_{2} \in$ $Z_{e}$. Then $u_{2}^{-1} u_{1} x_{1}=x_{2} v_{2} v_{1}^{-1}$. But $u_{2}^{-1} u_{2} x_{1} \in \overline{B^{-}}$and $x_{2} v_{2} v_{1}^{-1} \in \bar{B}$ and by [12, Proposition 27.2] $B^{-} \cap \bar{B}=\bar{T}=Z$ (since in any representation $\rho$ of $G$ we can put $\rho\left(B^{-}\right)$ and $\rho(B)$ in upper and lower triangular form, respectively, by choosing a suitable basis of weight vectors). Hence, $u_{2}^{-1} u, x, \in Z$. Letting $x_{1}=e t, t \in T$, we obtain also $u_{2}^{-1} u_{1} e \in Z$. By [19, I, Theorem 1.1], we may think of $E$ as a closed submonoid of End $_{k}\left(k^{n}\right)$ for some $n>0$, and in such a way that $Z$ consists of diagonal matrices and $B^{-}$consists of upper-triangular matrices [12, Proposition 15.4 and Theorem 17.6]. But $u_{2}^{-1} u_{1} e$ is now represented by a diagonal matrix with the same rank and eigenvalues as $e$. Further, $\left(u_{2}^{-1} u_{1} e\right) e=\left(u_{2}^{-1} u_{1} e\right)$. It follows that $u_{2}^{-1} u_{1} e=e$. But we have noted that (4.3) applies also to $B_{u}^{-} \rightarrow B_{u}^{-} e$ and thus $u_{2}^{-1} u_{1}=1$.

Similarly, $v_{1}=v_{2}$ and thus, $x_{1}=x_{2}$ as well. This proves

$$
m: B_{u}^{-} \times Z_{e} \times B_{u} \rightarrow E
$$

is injective. But from [12, Proposition 28.5] we know that $m$ is also birational. Hence, by ZMT it is actually an open embedding, since $E$ is normal.

(4.5) Corollary (Extension Principle). Let $E$ be reductive and normal, and suppose $\alpha: G \rightarrow E^{\prime}$ and $\beta: Z \rightarrow E^{\prime}$ are morphisms of algebraic monoids with $\left.\alpha\right|_{T}=\left.\beta\right|_{T}$. Then there exists a unique morphism $\varphi: E \rightarrow E^{\prime}$ such that $\left.\varphi\right|_{G}=\alpha$ and $\left.\varphi\right|_{T}=\beta$.

Proof. By the codim 2 condition (2.1) it suffices to extend $\alpha$ to a morphism $\gamma$ : $U \rightarrow E^{\prime}$, where $U \subseteq E$ is any open set with $\operatorname{codim}_{E}(E \backslash U) \geqslant 2$. 
Let $V \subseteq E \backslash G$ be any irreducible component. By [29, Theorem 4.3] there exists a maximal idempotent $e \in V \cap I(Z)$. Hence it suffices to find, for such an $e$, an open set $U_{e} \subseteq E$ with $e \in U_{e}$ so that $\alpha$ extends to a morphism $\varphi_{e}: U_{e} \cup G \rightarrow E^{\prime}$.

Let $U_{e}=B_{u}^{-} \times Z_{e} \times B_{e}$ be as in Theorem (4.4) and $m: U_{e} \rightarrow E$ the associated open embedding. (We can assume $e \in \Lambda(B)$ for some $B$ since $I(Z)=\bigcup_{T \subseteq B} \Lambda(B)$.)

Define $\varphi_{e}: U_{e} \rightarrow E^{\prime}$ by $\varphi_{e}(x, y, z)=\alpha(x) \beta(y) \alpha(z)$. This is the desired extension that completes the proof.

5. The construction. In this section we construct for each polyhedral root system $(X, \Phi, C)$ a semisimple monoid $E$ such that $(X(T), \Phi(T), X(Z) \cong(X, \Phi, C))$. The major techniques for the construction have been assembled in $\S 3$.

To simplify the proof of (5.1), we shall impose a mild constraint on the underlying group of units. However, all results are valid and will be stated for any reductive group with one-dimensional center. This "loan" will be paid back (with interest!) in $\S 7$.

Let $G=G_{0} \times k^{*}$ be the reductive, algebraic group where $G_{0}$ is semisimple and simply connected. This is our constraint. It is needed to simplify the proofs for monoids in characteristic $p>0$.

Suppose $(X, \Phi, C)$ is a polyhedral root system of type $G$.

(5.1) Proposition. There exists a semisimple algebraic monoid $E$, with unit group $G$, such that $(X(T), \Phi, X(Z)) \cong(X, \Phi, C)$. (Here, $T, \Phi$ and $Z$ have the usual meanings.)

Proof. Since $G_{0}$ is simply connected, there exists a completely reducible, schematically faithful representation of $G[3, \mathrm{~A}$, Theorem 6.4]. The same is true for $G=G_{0} \times k^{*}$ since all rational representations of $k^{*}$ are completely reducible [12, §16.4].

Fix an identification $(X(T), \Phi(T)) \equiv(X, \Phi)$. We may then regard $C$ as a convex subset of $X(T)$ stable under the action of the Weyl group.

By Gordon's lemma [14, p. 7], $C$ is finitely generated by a subset

$$
S=\left\{\left(\lambda_{i}, n_{i}\right)\right\}_{i=1}^{s} \subseteq C \subseteq X(T)=X\left(T_{0}\right) \oplus \mathbf{Z} .
$$

We may assume further that $S$ is $W$-stable.

Now each $\alpha \in X\left(T_{0}\right)$ is $W$-conjugate to exactly one dominant weight $\lambda \in X^{+}\left(T_{0}\right)$ (2.2). Let

$$
S^{+}=\{(\lambda, n) \in S \mid \lambda \text { is dominant }\} .
$$

Define $\rho_{n}^{\lambda}: G \rightarrow \operatorname{Gl}(V)$ for $(\lambda, n) \in S^{+}$as in (3.4).

By (3.5), $\Phi\left(\rho_{n}^{\lambda}\right) \subseteq C$ since $C$ is convex. Thus, if we let

$$
\varphi=\bigoplus_{(\lambda, n) \in S^{+}} \rho_{n}^{\lambda}: G \rightarrow \mathrm{Gl}_{t}(k), \quad t=\sum_{(\lambda, n) \in S^{+}} \operatorname{dim} \rho_{n}^{\lambda},
$$

we obtain $\Phi(\varphi) \subseteq C$ since $\Phi(\varphi)=\bigcup_{(\lambda, n) \in S^{+}} \Phi\left(\rho_{n}^{\lambda}\right)$, and $X(\overline{\varphi(T)})=C$ (by (3.3)) since $\Phi(\varphi)$ generates $C$ as a monoid. Hence, if $E_{1}=\overline{\varphi(G)} \subseteq$ End $_{k}\left(k^{t}\right)$, then $\left(X\left(T_{1}\right), \Phi\left(T_{1}\right), X\left(Z_{1}\right)\right) \cong(X, \Phi, C)$. But $E_{1}$ may not be normal. So let $E \underset{\eta}{\rightarrow} E_{1}$ be 
the normalization of $E_{1}$ (2.1). Restricting $\eta$ to maximal $D$-submonoids we obtain $\left.\eta\right|_{Z}: Z \rightarrow Z_{1}$, which is finite and birational. Since $Z_{1}$ is normal $(C$ is $),\left.\eta\right|_{Z}$ is an isomorphism. Thus, $X(Z)=X\left(Z_{1}\right)=C$. This concludes the construction.

ReMARK. If $G=\mathrm{PGl}_{2}(k) \times k^{*}$ and $\operatorname{char}(k)=2$, then $G$ has no completely reducible, schematically faithful representation. In fact, if $\rho: \operatorname{PGl}_{2}(k) \rightarrow \mathrm{Gl}(V)$ is any irreducible representation, then

$$
\operatorname{Ker}(\rho) \supseteq\left\{\left[\begin{array}{ll}
a & b \\
c & d
\end{array}\right] \mid \begin{array}{c}
a^{2}=d^{2}=1, a d-b c=1, \\
b^{2}=c^{2}=0
\end{array}\right\}
$$

as schemes.

6. Uniqueness. Now that we have constructed a semisimple monoid for each polyhedral root system, it remains for us to show that distinct monoids have distinct polyhedral root systems, so that our construction in $\$ 5$ is both complete and discriminating. The main result of this section (Theorem (6.5)) is deduced from the conclusions of $\S 4$.

We fix notation.

Let $E$ be a semisimple, algebraic monoid with maximal $D$-submonoid $Z \subseteq E$. ( $X, \Phi, C$ ) will denote a polyhedral root system of type $G=G(E)$.

If $E$ is semisimple, we have yet to deduce that $(X(T), \Phi(T), X(Z))$ is a polyhedral root system. However, if, for the moment, we let $C(Z)=\{x \in X(T) \mid n x \in$ $X(Z)$ for some $n>0\}$, then by the proof of (3.7), $(X(T), \Phi(T), C(Z))$ is a polyhedral root system. (We show in (6.4) that $X(Z)=C(Z)$.) $X(Z) \rightarrow C(Z)$ corresponds, by duality, to the normalization morphism $\tilde{Z} \rightarrow Z(2.1)$. We shall refer to $(X(T), \Phi(T), C(Z))$ as the polyhedral root system associated with $E$.

The basic idea behind our arguments is quite simple and is outlined here.

Given $E$, let $(X, \Phi, C)$ be the associated polyhedral root system. By (5.1) there exists a semisimple monoid $E_{1}$ with $\left(X\left(T_{1}\right), \Phi\left(T_{1}\right), X\left(Z_{1}\right)\right) \cong(X, \Phi, C)$ (so $X\left(Z_{1}\right)=$ $C(Z)$ ). It becomes clear that we may construct a commutative diagram

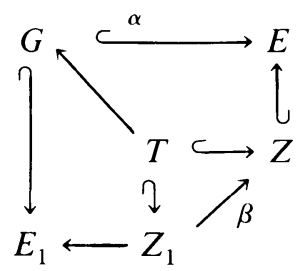

where $\beta$ is normalization. By (4.5) it follows that we may solve the lifting problem

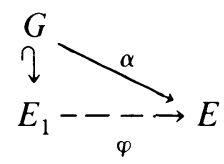

for $\varphi$. It then follows from general properties of reductive monoids and ZMT, that $\varphi$ is an isomorphism.

The remainder of this section is a record of the proofs of these assertions. The reader is reminded that most of the work has already been done in $\S \S 4$ and 5 . 
(6.1) LeMMA. Let $E$ and $(X(T), \Phi(T), C(Z))$ be as above and let $E_{1}$ be the monoid of type $G=G(E)$ with polyhedral root system $(X(T), \Phi(T), C(Z)$ ) constructed in (5.1). Then there exists a commutative diagram

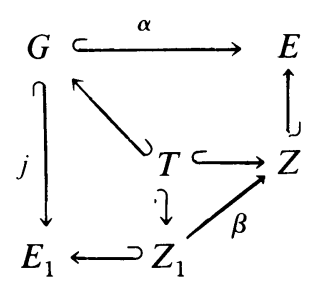

where $\beta: Z_{1} \rightarrow Z$ is the normalization morphism.

Proof. From (5.1) we obtain the diagram:

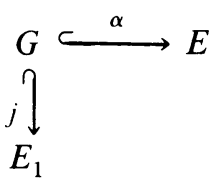

The construction of $E_{1}$ in the proof of (5.1) identifies the image of $X\left(Z_{1}\right) \rightarrow_{j^{*}} X(T)$ with $C(Z)$. Thus, we have

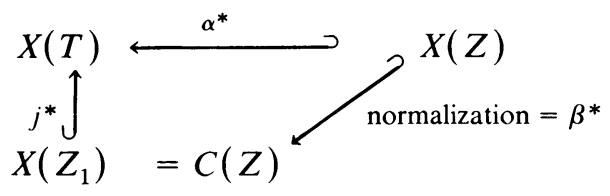

which is commutative.

If we dualize the diagram (*) it fits into the diagram ( $\dagger$ ) to yield the commutative diagram (1).

(6.2) Corollary. Let $E$ and $E_{1}$ be as in (6.1). Then there exists a morphism $\varphi$ : $E_{1} \rightarrow$ E such that $\left.\varphi\right|_{G}=\alpha$ and $\left.\varphi\right|_{Z_{1}}=\beta$.

Proof. Since diagram (1) of (5.1) is commutative, it follows that $\left.\alpha\right|_{T}=\left.\beta\right|_{T}$. Thus, by (4.5) there exists such a morphism $\varphi$.

(6.3) COROllaRY. The morphism $\varphi$ of (6.2) is an isomorphism.

Proof. $\varphi$ is dominant and $\left.\varphi\right|_{Z}$ is finite. It follows that $\varphi^{-1}(0)$ is a nilpotent ideal of $E_{1}$ since if $x \in \varphi^{-1}(0)$ is not nilpotent there is an idempotent $0=e \in I(E)$ such that $y x^{n}=e$ for some $n>0$ and some $y \in E[19$, I, Corollary 1.4]. But then

$$
0=\varphi(y) \varphi\left(x^{n}\right)=\varphi\left(y x^{n}\right)=\varphi(e)
$$

This is impossible, since $e$ is conjugate to a nonzero idempotent of $Z_{1}$ [20, Theorem $1.8]$ and $\left.\varphi\right|_{Z_{1}}: Z_{1} \rightarrow Z$ is a finite morphism. Thus, since the zero element is unique, $\left(\left.\varphi\right|_{Z_{1}}\right)^{-1}(0)=\{0\}$.

On the other hand, $E_{1}$ is a regular monoid (2.3) and therefore any ideal $\left(\varphi^{-1}(0)\right)$ must contain idempotents. Hence $\varphi^{-1}(0)=\{0\}$. By NAK (2.1) $\varphi$ is a finite morphism. But now by ZMT (2.1) $\varphi$ is an isomorphism since $E$ is normal and $\varphi$ is surjective. 
(6.4) Corollary. Let $E$ be semisimple. Then $Z$ is normal. In particular, $(X(T), \Phi(T), X(Z))$ is a polyhedral root system (i.e. $X(Z)=C(Z))$.

Proof. $Z_{1}$ is normal by construction and $\left.\varphi\right|_{Z_{1}}: Z_{1} \rightarrow Z$ is an isomorphism by (6.3).

(6.5) TheOREM. The correspondence $E \rightarrow(X(T), \Phi(T), X(Z))$ determines a bijection between the set of isomorphism classes of semisimple, algebraic monoids and the set of isomorphism classes of polyhedral root systems.

Proof. By (3.7) and (6.4) $(X(T), \Phi(T), X(Z))$ is a polyhedral root system and by (6.3), any semisimple monoid with this polyhedral root system is isomorphic to $E$. By (5.1) every polyhedral root system is associated with some $E$.

7. Tidying up. In this section we finish the proof of (5.1) ("nonsimply connected" case) and introduce and clarify a few notions that will be useful in $\S 8$ when we deduce some corollaries of the classification theory. Since the subsections of this section are independent, the reader may wish to skip to $\S 8$ and refer to these results as they are needed.

(7.1) Semisimple monoids. Recall from (5.1) that we have stated our results for monoids with reductive unit group $G$ with $\operatorname{dim} Z G=1$; but the proof was given only for $G=G_{0} \times k^{*}$ with $G_{0}$ semisimple and simply connected.

Let $G$ be reductive with $\operatorname{dim} Z G=1$ and let $G_{0}=(G, G)^{\prime}$ (universal cover). Then $(Z G)^{0} \cong k^{*}$ and we have a central isogeny

$$
j: G_{0} \times k^{*} \rightarrow G, \quad(g, t) \rightarrow g t,
$$

that is, $j$ is finite and dominant, and $\operatorname{Ker}(j) \subseteq Z\left(G_{0} \times k^{*}\right)$.

(7.1.1) LEMMA. Let $j$ be as above and suppose $i: G=G(E) \subseteq E$ for some semisimple monoid $E$. Then there exists a semisimple monoid $E^{\prime}$ with unit group $i^{\prime}: G_{0} \times k^{*} \rightarrow E^{\prime}$ and a finite morphism $\varphi: E^{\prime} \rightarrow E$ of algebraic monoids such that $\varphi \circ i^{\prime}=i \circ j$. Furthermore, $E^{\prime}$ is unique.

PROOF. The detailed argument has been carefully written down in [27, §3.2], but is easily reconstructed from the following sketch:

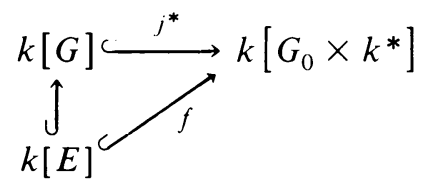

Let $R \subseteq k\left[G_{0} \times k^{*}\right]$ be the integral closure of $k[E]$ in $k\left[G_{0} \times k^{*}\right]$. As $f$ is a map of bialgebra, one can deduce that $R$ is also a bialgebra since $R \otimes R \rightarrow k\left[G_{0} \times k^{*}\right] \otimes$ $\left[G_{0} \times k^{*}\right]$ is integrally closed. Furthermore, $R$ is finitely generated since $j^{*}$ is a finite morphism. Hence, by [7, Proposition 1.6, Chapter 2], $R=k\left[E^{\prime}\right]$ for some algebraic monoid $E^{\prime}$ with $i^{\prime}: G_{0} \times k^{*} \rightarrow E^{\prime}$ dual to the inclusion of bialgebra $R \subseteq k\left[G_{0} \times k^{*}\right]$. $i^{\prime}$ is an open immersion because localization commutes with integral closure [1, Proposition 5.12]. $\varphi$ is the finite morphism dual to the inclusion $k[E] \subseteq R$. Clearly, $E^{\prime}$ is unique. 
(7.1.2) Discussion (7.1.1) gives us a canonical correspondence

semisimple monoids with group $G \rightarrow$ semisimple monoids with group $G_{0} \times k^{*}$.

Let $(X, \Phi, C)$ be the polyhedral root system of $E$. Corresponding to $\varphi$ we have an induced map

$$
\varphi^{*}:(X, \Phi, C) \rightarrow\left(X^{\prime}, \Phi^{\prime}, C^{\prime}\right) .
$$

$\varphi^{*}$ is the map induced from $\left.\varphi\right|_{Z^{\prime}}: Z^{\prime} \rightarrow Z . \varphi^{*}(\Phi)=\Phi^{\prime}$ since $\operatorname{ker}(\varphi) \subseteq Z\left(G_{0} \times k^{*}\right)$ and $C^{\prime}=\left\{\chi \in X^{\prime} \mid n \chi \in \varphi^{*}(C)\right.$ for some $\left.n>0\right\}$ since $C^{\prime}$ is normal.

(7.1.3) LemMa. Let $E^{\prime}$ be a semisimple monoid with unit group $i^{\prime}: G_{0} \times k^{*} \rightarrow E^{\prime}$ and let $j: G_{0} \times k^{*} \rightarrow G$ be as in (7.1.1). Then there exists a semisimple monoid $E$ with unit group $i: G \rightarrow E$ and a finite dominant morphism $\varphi: E^{\prime} \rightarrow E$ such that $\varphi \circ i^{\prime}=i \circ j$.

Proof. Let $K=\operatorname{ker}(j)$. Then $K$ is a finite $D$-group [34, §2.2]. Assume $(\operatorname{deg}(j), \operatorname{char}(k))=1$. Otherwise, our argument requires modifications that depend on the techniques of [34]. We leave that part to the reader.

By our assumption, $j$ is separable, and further, $G=\left(G_{0} \times k^{*}\right) / K . K$ acts on $E^{\prime}$ also by the rule

$$
K \times E^{\prime} \rightarrow E^{\prime}, \quad(t, x) \rightarrow t x .
$$

Since $E^{\prime}$ is affine, the quotient variety $E=E^{\prime} / K$ exists and is affine [16, §12]. (Here $k[E]=k\left[E^{\prime}\right]^{K} \hookrightarrow_{\varphi^{*}} k\left[E^{\prime}\right]$.) Let $\varphi: E^{\prime} \rightarrow E$ be the quotient morphism. $E$ is a monoid since in the diagram

$$
\begin{array}{lll}
E^{\prime} \times E^{\prime} \stackrel{m}{\rightarrow} & E^{\prime} \\
\varphi \times \varphi \downarrow & & \downarrow \varphi \\
E \times E- & & \rightarrow
\end{array}
$$

$\varphi \circ m$ is constant on the fibres of $\varphi \times \varphi$, which is a quotient morphism. (See [30, §4.3] for more discussion of the same argument applied to a special case.) Thus we obtain the following commutative diagram of algebraic monoids:

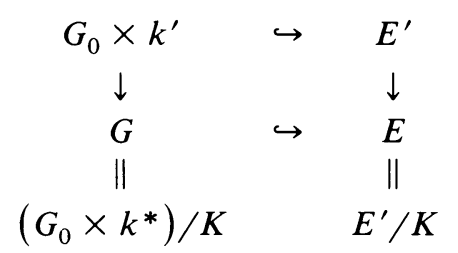

$E$ is normal by [28, Lemma 2.2] and thus, semisimple. We leave the remaining details to the reader.

(7.1.4) Discussion. (7.1.3) yields a canonical correspondence

semisimple monoids with group $G_{0} \times k^{*} \rightarrow$ semisimple monoids with group $G$.

If $\left(X^{\prime}, \Phi^{\prime}, C^{\prime}\right)$ is the polyhedral root system of $E^{\prime}$ and $\left.j\right|_{T}: T^{\prime} \rightarrow T=T^{\prime} / K$, then $(X, \Phi, C)$ is the polyhedral root system of $E$, where $X=X(T)=X\left(T^{\prime} / K\right) \subseteq \subseteq^{*}$ $X\left(T^{\prime}\right), \Phi=\Phi^{\prime}$ and $C=C^{\prime} \cap X(T)$. 
(7.1.5) TheOREM. Let $(X, \Phi, C)$ be a polyhedral root system of type $G$. Then there exist a semisimple monoid $E$, unique to within an isomorphism, with polyhedral root system $(X, \Phi, C)$.

Proof. Let $j: G_{0} \times k^{*} \rightarrow G$ be as in (7.1.1) and let $\varphi=\left.j\right|_{T}: T^{\prime} \rightarrow T$ be the map induced on maximal tori. Define a polyhedral root system of type $G_{0} \times k^{*}$ as follows:

$$
\begin{aligned}
& X^{\prime}=X\left(T^{\prime}\right) \subseteq X(T), \\
& \Phi^{\prime}=\Phi(T) \subseteq X\left(T^{\prime}\right) \quad(\text { since } \operatorname{ker}(j) \text { is central), } \\
& C^{\prime}=\left\{x \in X^{\prime} \mid n x \in C \text { for some } n>0\right\}
\end{aligned}
$$

(the "normalization" of $(X, \Phi, C)$ in $\left.\left(X^{\prime}, \Phi^{\prime}\right)\right)$. It is simple to verify that $\left(X^{\prime}, \Phi^{\prime}, C^{\prime}\right)$ is a polyhedral root system of type $G_{0} \times k^{*}$. Thus, by (5.1), there exists a unique, semisimple monoid $E^{\prime}$ with polyhedral root system $\left(X^{\prime}, \Phi^{\prime}, C^{\prime}\right)$ and unit group $i^{\prime}$ : $G_{0} \times k^{*} \rightarrow E^{\prime}$. But now (7.1.3) applies and we have a commutative diagram

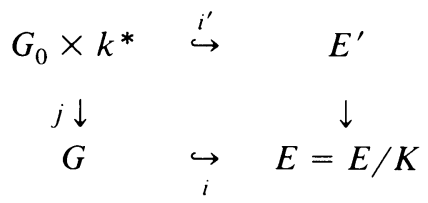

where $K=\operatorname{ker}(j)$.

By (7.1.4), the polyhedral root system of $E$ is $\left(X\left(T^{\prime} / K\right), \Phi, C^{\prime} \cap X\left(T^{\prime} / K\right)\right)$. But $X\left(T^{\prime} / K\right)=X(T)$ and by (*), we must have $C^{\prime} \cap X(T)=C$ since $C$ is normal (2.3.2). Thus, $(X, \Phi, C)$ is the polyhedral root system of $E$. The uniqueness of $E$ follows from (7.2) and (7.4) since those two correspondences are inverse to each other (by the uniqueness statements of (7.1.1) and (7.1.3)).

(7.2) Monoids with reductive unit group. We are studying only normal monoids with unit group $G$, isogenous to $G_{0} \times k^{*}\left(G_{0}\right.$ semisimple). The reader may wonder if our methods apply to any normal, reductive monoid. This is almost certainly true. For example, Theorem (4.4) has been proved for any reductive monoid. However, I have chosen to consider only semisimple monoids mainly for the sake of exposition. Although the general classification should now be a routine exercise (given [33, Theorem 11.4.3]), this paper could not benefit from the resulting technical complications. In (7.3) we consider the special case that is needed for refining the classification of semisimple monoids.

(7.3) Further refinements of the classification. The purpose of this section is to resolve the ambiguity inherent in the association $G \rightarrow(X(T), \Phi(T))$ if $G$ is reductive with $\operatorname{dim} Z G=1$.

The problem is that one cannot reconstruct the original Weyl group action on $X(T)$, as one does in the semisimple case, using only the configuration of $\Phi$ in $X$ (see [12, Appendix A1]). We have managed to avoid this problem by insisting that the Weyl group can be chosen to stabilize a polyhedral cone (see (3.6) and comment (2) following it). But now we would like to start with $(X(T), \Phi(T))$ of type $G$ and then classify all possible, theoretically distinct polyhedral root systems (of type $G$ ) in the most efficient way. Thus, we are committed to finding a more rigid object than $(X(T), \Phi(T))$. 
(7.3.1) Definition. (a) A root system of (semisimple) corank one is a triple $(X, \Phi, L)$, where:

(i) $\Phi \subseteq X$ is finite, does not contain 0 and spans a subgroup $(\Phi)$ of corank one.

(ii) $L \subseteq X$ is a subgroup of rank one such that $(\Phi) \cap L=(0)$.

(iii) $\left(X_{0}, \Phi\right)$ is a root system (in the sense of $\S 3$ ), where $X_{0}=\{\chi \in X \mid n \chi \in(\Phi)\}$.

(iv) The reflections $\sigma_{\alpha}: X_{0} \rightarrow X_{0}$ of $\left(X_{0}, \Phi\right)$ can be extended to $X$ such that $L \subseteq X^{\sigma_{\alpha}}$ for all $\alpha \in \Phi$.

(b) We say that $(X, \Phi, L)$ is oriented if we have chosen a generator $\chi$ of $L$. We let $L^{+}=\{0, \chi, 2 \chi, \ldots\}$.

REMARKS. (1) There are two important examples of root systems of corank one. (The second one is canonically oriented.)

(a) Let $G$ be reductive such that $\operatorname{dim} Z G=1$. Then it is easy to check that $(X(T), \Phi(T), X(G))$ is a root system of corank one.

(b) Further, if $G \subseteq E$ is a semisimple algebraic monoid, then $\mathbf{N} \cong X(E) \subseteq X(G)$ $\cong \mathbf{Z}$ determines a canonical orientation.

(2) It is a straightforward exercise (requiring only the results of [12]) in "central isogeny" to prove that the correspondence

$$
G \rightarrow(X(T), \Phi(T), X(G))
$$

induces a canonical bijection, to within an isomorphism, between the class of reductive groups with one-dimensional center and the class of root systems of corank one. Alternatively, we could deduce the result from $[33, \S 11.4]$ once the appropriate translation between (7.3.1) and the terminology of [33] is made.

Given $(X, \Phi, L)$ of type $G$, let

$$
\operatorname{Aut}^{+}(X, \Phi, L)=\left\{\lambda \in \operatorname{Aut}(X, \Phi, L)|\lambda|_{L}=\mathrm{id}\right\}
$$

and

$$
\Gamma=\Gamma_{\Delta}=\left\{\lambda \in \operatorname{Aut}^{+}(X, \Phi, L) \mid \lambda(\Delta)=\Delta\right\},
$$

where $\Delta \subseteq \Phi$ is any base (2.2). Then as in [11, §12.2]

$$
\operatorname{Aut}^{+}(X, \Phi, L) \cong W \rtimes \Gamma \quad \text { (semidirect product). }
$$

We can now present a more vivid picture of the classification of semisimple monoids.

Let $(X, \Phi, C)$ be a polyhedral root system such that $X=X(T), \Phi=\Phi(T)$ and $\chi=\sum \chi_{i} \in L^{+}\left(\left\{\chi_{i}\right\}\right.$ are the fundamental generators (3.1)). We can always arrange this because if $\chi \in L^{-}$, then we have an isomorphism

$$
(X, \Phi, C) \cong(X, \Phi,-C), \quad x \rightarrow-x,
$$

and $(X, \Phi,-C)$ is of the desired type.

Let

$$
\mathscr{E}=\left\{C \subseteq X \mid(X, \Phi, C) \text { is a polyhedral root system and } \chi \in L^{+}\right\} .
$$

$\Gamma$ acts on $\mathscr{E}$ by the rule

$$
\Gamma \times \mathscr{E} \rightarrow \mathscr{E}, \quad(\lambda, C) \rightarrow \lambda(C)
$$


(7.3.2) Proposition. $\mathscr{E} / \Gamma$, the orbit set, classifies polyhedral root systems of type $G$, up to isomorphism.

We omit the (simple) proof.

Let

$$
\mathscr{B}=\left\{\begin{array}{c}
A \text { is a sub-bialgebra, } \\
A \subseteq k[G] \mid A \text { is finitely generated, } A_{0}=k, \\
\chi \in A \text { and } A[1 / \chi]=k[G]
\end{array}\right\} .
$$

Here $A_{0}=A^{Z G^{0}}$ ( $Z G^{0}$ acts on $A$ since $A \subseteq k[G]$ is a sub-bialgebra) and $\chi \in X(G)$ is a fixed, nontrivial character which gives the orientation.

(7.3.3) COROLlaRY. There is a canonical one-to-one correspondence $\mathscr{E} \cong \mathscr{B}$.

Proof. From (6.5), any $(X, \Phi, C)$ yields an $E$, semisimple, with $G \subseteq E$ and $X(Z)=C$. Thus, $k[E] \in \mathscr{B}$.

Conversely, if $A \in \mathscr{B}$, then $E=\operatorname{Spec}(A)$ is a semisimple monoid of type $G$ (with orientation given by $\chi)$. Hence $(X(T), \Phi(T), X(Z))$ is a polyhedral root system of the desired type and so $X(Z) \in \mathscr{E}$.

The remaining details are left to the reader.

REMARK. $\mathscr{B}$ can be thought of as the set of isomorphism classes of pairs $(i, E)$, where $i: G \rightarrow E$ is a preferred embedding of $G=G(E)$.

(7.4) Anti-involutions.

Proposition. Every reductive group $G$ admits an anti-involution $\tau: G \rightarrow G$ such that $\tau(t)=t$ for all $t \in T$.

Proof. There exists an automorphism $\sigma: G \rightarrow G$ with the property $\sigma(t)=t^{-1}$ for all $t$, by Theorem 11.4 .3 of [33] (suitably translated) applied to

$$
(X, \Phi) \rightarrow(X, \Phi), \quad x \rightarrow-x .
$$

Let $\tau_{1}=\sigma \circ i$, where $i(g)=g^{-1}$ for all $g \in G$. Then $\tau_{1}$ is an antiautomorphism with $\tau_{1}(t)=t$ for all $t \in T$. By Theorem 27.4 of [12] $\tau_{1}^{2}=\operatorname{int}(g) \in \operatorname{Int}(G)$. But now $g \in C_{G}(T)$, so by Corollary A of $[12, \S 26.2] g \in T$. Let $h \in T$ be such that $h^{2}=g$. Then $\tau=\operatorname{int}\left(h^{-1}\right) \circ \tau_{1}$ is the desired anti-involution.

The most familiar example is $\tau(A)={ }^{t} A, A \in \mathrm{Gl}_{n}(k)$. What is most surprising is that $\tau$ can be extended over any semisimple monoid $E$ with $G(E)=G$ (see (8.2) for the proof).

8. Some consequences. The purpose of this section is to record some of the immediate consequences of the classification (6.5).

(8.1) Birational morphisms. A well-known property of algebraic groups [34, §14.3] is the following:

If $\varphi: G \rightarrow H$ is a birational morphism of irreducible algebraic groups, then $\varphi$ is an isomorphism.

This property fails for reductive monoids but nonetheless we can arrive at a definitive classification of birational morphisms at least in the semisimple case. 
The most familiar (reductive) example is

$$
k^{2} \rightarrow k^{2}, \quad(x, y) \rightarrow(x, x y) .
$$

Here, $k^{2}$ has the usual multiplicative structure.

Semisimple examples are more complicated but the theory is very satisfying. Given semisimple algebraic monoids $E_{1}$ and $E_{2}$ with unit group $G$ we shall solve the two following problems:

(1) Under what conditions does there exist a birational morphism $\psi: E_{1} \rightarrow E_{2}$ ?

(2) If the conditions in (1) are satisfied, find a numerical identification of the set of conjugacy classes of these $\psi$.

We use the results of (7.3). $\Gamma, \mathscr{E}$ and $\mathscr{B}$ will have the same meanings.

THEOREM. Let $E_{1}$ and $E_{2}$ be semisimple algebraic monoids with unit group $G$, and fix inclusions $i_{j}: G \rightarrow E_{j}, j=1,2$. Let $T \subseteq G$ be a maximal torus and let $C_{i}=X\left(Z_{i}\right) \subseteq{ }_{i_{j}^{*}}$ $X=X(T)$.

(a) There exists a birational morphism $\psi: E_{1} \rightarrow E_{2}$ such that $\psi \circ i_{1}=i_{2}$ if and only if $C_{2} \subseteq C_{1}$.

(b) There exists a birational morphism $\varphi: E_{1} \rightarrow E_{2}$ if and only if there exists $\lambda \in \Gamma$ such that $\lambda\left(C_{2}\right) \subseteq C_{1}$.

(c) The set of conjugacy classes of birational morphisms from $E_{1}$ to $E_{2}$ is in one-to-one correspondence with $\left\{\lambda \in \Gamma \mid \lambda\left(C_{2}\right) \subseteq C_{1}\right\}$.

Proof. (a) The "if" part follows directly from (4.5); we have $\beta: Z_{1} \rightarrow E_{2}$, extending $i_{2}: T \rightarrow E_{2}$ since $C_{2} \subseteq C_{1}$.

Conversely, if we have $\varphi: E_{1} \rightarrow E_{2}$ with $\varphi \circ i_{1}=i_{2}$, then $\varphi: Z_{1} \rightarrow Z_{2}$ induces $\varphi^{*}\left(C_{2}\right) \subseteq C_{1}$. Under our identification $\left(i_{j}: C_{j} \subseteq X(T)\right)$ we have $C_{2} \subseteq C_{1}$.

(b), (c) Consider the diagram

$$
\begin{array}{ccc}
G & \stackrel{i_{1}}{\hookrightarrow} & E_{1} \\
\bar{\lambda} \downarrow & & \\
G & \stackrel{\hookrightarrow}{i_{2}} & E_{2}
\end{array}
$$

where $\bar{\lambda}: G \rightarrow G$ is any lifting of $\lambda: X(T) \rightarrow X(T)$ to an automorphism of $G$ (as in Theorem' 32 of [12] slightly generalized). By our assumptions $\bar{\lambda}^{*} \circ i_{2}^{*}\left(C_{2}\right) \subseteq i_{1}^{*}\left(C_{1}\right)$, and again we may apply (4.5).

Conversely, if we have $\varphi: E_{1} \rightarrow E_{2}$, then conjugating $\varphi$, if necessary, we may assume that $\varphi(T)=T$ since all maximal tori are conjugate. Hence,

$$
\left.\varphi^{*}\right|_{X(T)} \in \operatorname{Aut}^{+}(X(T), \Phi(T), X(G)) \cong W \rtimes \Gamma
$$

by the results of (7.3). So we may assume $\left.\varphi^{*}\right|_{(X(T))}=\lambda \in \Gamma$ by conjugating with some element of $W$ if necessary. Hence, given our identification of $\left(X_{1}, \Phi_{1}\right)$ and ( $\left.X_{2}, \Phi_{2}\right)$ we have found $\lambda \in \Gamma$ such that $\lambda\left(C_{2}\right) \subseteq C_{1}$.

Remark. The possibilities for $\Gamma$, and hence $\left\{\lambda \in \Gamma \mid \lambda\left(C_{2}\right) \subseteq C_{1}\right\}$, are severely restricted. See [11, §12.2] for the (finite) upper bound in each case. 
(8.2) Antiautomorphism. Another well-known property of groups, so tautologous that it should never be mentioned, is that any group $G$ (in any category) is anti-isomorphic to itself:

$$
G \rightarrow G, \quad x \rightarrow x^{-1}
$$

It is surprising though, to discover that any semisimple, algebraic monoid $E$ is anti-isomorphic to itself, and in a particularly nice way.

THEOREM. Let $E$ be a semisimple algebraic monoid and $T \subseteq G(E)$ a maximal torus. There exists a morphism $\tau: E \rightarrow E$ of varieties such that

(i) $\tau^{2}=\mathrm{id}$,

(ii) $\tau(x y)=\tau(y) \tau(x)$ for all $x, y \in E$,

(iii) $\tau(t)=t$ for all $t \in T$.

Furthermore,

(iv) $\tau\left(U_{\alpha}\right)=U_{-\alpha}$ for all $\alpha \in \Phi(T)$ and in particular

(v) $E \cong E^{\mathrm{op}}$, the opposite monoid of $E$.

Proof. By Proposition (7.4) there exists a morphism of varieties $\tau: G \rightarrow G$ satisfying properties (i)-(iii).

We first deduce (iv) and (v) assuming $\tau$ extends to $E$.

(iv) Let $\alpha \in \Phi, T_{\alpha}=\left(T^{\sigma_{\alpha}}\right)^{0}$ and $G_{\alpha}=\left(C_{G_{\alpha}}(T), C_{G_{\alpha}}(T)\right)$. Then $G_{\alpha} \cong \mathrm{Sl}_{2}(k)$ or $\operatorname{PGl}_{2}(k)[12, \S 32.3]$ and $\tau\left(G_{\alpha}\right)=G_{\alpha}$. But in this case it is well known that $\tau$ must be either $T=$ transpose, or $\operatorname{int}(t) \circ T$, where $t^{2} \in Z G_{\alpha}$. Either way $\tau\left(U_{\alpha}\right)=U_{-\alpha}$.

(v) is obvious.

So we are left with extending $\tau: G \rightarrow G$ to $\tau: E \rightarrow E$.

Consider the solid arrow diagram

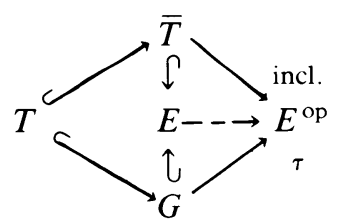

which is commutative because $\left.\tau\right|_{T}=\mathrm{id}$, and is a diagram of algebraic monoids because $\bar{T}$ is commutative. By (4.5) the dotted arrow exists and we have a morphism $\tau: E \rightarrow E^{\mathrm{op}} . \tau$ is an anti-isomorphism since $\tau^{2}=1$ on $G$, and thus on $E$ since $G \subseteq E$ is dense.

(8.3) Monoids with unique minimal $\mathscr{J}$-class. A key factor in the structure theory of algebraic monoids is the set of $\mathscr{J}$-classes. This notion has been introduced by Green [9] in the study of abstract semigroups and is now an important part of a general theory (known as Green's relations). We shall not need the original definition because in our context there is a much simpler formulation due to M. Putcha [21].

We begin with a summary of some results from [21 and 25]. $E$ denotes a reductive, algebraic monoid. Let $Z \subseteq E$ be a maximal $D$-submonoid. Then $E=\cup_{e \in I(Z)} G e G$ since $E$ is regular (2.3). This union is not disjoint but $G e G=G f G$ if and only if $e=$ wew $^{-1}$ for some $w \in N_{G}(T)$ [22, Proposition 3.1]. Hence, we may write

$$
E=\coprod_{e \in I(Z) / W} G e G .
$$


It turns out that $\mathscr{U}(E)=\{G e G\}$ is precisely the set of $\mathscr{J}$-classes ([22], taking into account that $E$ is regular) and we take this as our definition.

Suppose $E$ has a zero. Then we let

$$
\mathscr{U}_{1}(E)=\left\{\left.\alpha \in \mathscr{U}(E)\right|_{\text {nonzero idempotents }} ^{\alpha \cap I(Z) \text { consists of minimal }}\right\},
$$

the set of minimal g-classes.

Let $|S|$ denote the cardinality of the set $S$.

(8.3.1) Definition. The reductive monoid $E$, with 0 , is $\mathscr{g}$-irreducible if $\left|\mathscr{U}_{1}(E)\right|=1$.

By (1) this is equivalent to asserting that $W=N_{G}(T)$ acts transitively on the minimal idempotents of $Z$. Let $I_{1}(Z)$ denote the set of minimal, nonzero idempotents of $Z$.

(8.3.2) Lemma. Suppose E is g-irreducible and normal. Then $E$ is semisimple.

Proof. We must prove that $\operatorname{dim} Z G(E)=1$. Let $\rho: E \rightarrow \operatorname{End}_{k}(V)$ be a faithful representation which is a closed embedding with $\rho(0)=0_{v}[19$, I, Theorem 1.1] and let $e \in I(E)$ be any nonzero, minimal idempotent. By assumption, $\rho(e) \in \operatorname{End}(V)$ is also a nonzero idempotent. Let

$$
(0)=V_{0} \subseteq V_{1} \subseteq \cdots \subseteq V_{n}=V
$$

be a composition series for the $E$-module $V$. Since $\rho(e)$ is a nonzero, diagonalizable endomorphism of $V, \bar{\rho}(e)$, the map induced by $\rho(e)$ on $W=V_{i} / V_{i=1}$, is nonzero for some $0<i \leqslant n$. So consider the induced morphism $\bar{\rho}: E \rightarrow \operatorname{End}(W)$, with $\bar{\rho}(e) \neq$ $\rho(0)=0$. From (1) it follows that either

$$
\bar{\rho}^{-1}(0)=\{0\} \quad \text { or } \quad \bar{\rho}^{-1}(0) \supseteq G f G
$$

for some $0 \neq f \in I(Z)$. In the latter case we must have $E f E \subseteq \bar{\rho}^{-1}(0)$ since $G f G \subseteq E f E$ is dense. $E f E \cap I_{1}(Z) \neq \varnothing$ since $Z f Z=Z f$ and $f e_{1}=e_{1}$ for some minimal $e_{1} \in$ $I_{1}(Z)$. But all minimal idempotents of $Z$ are conjugate and thus $e \in E f E$ since $E f E$ is a two-sided ideal. This contradiction proves that $\bar{\rho}^{-1}(0)=\{0\}$ is the only possibility. Thus it follows from NAK (2.1) that

$$
\bar{\rho}: E \rightarrow \operatorname{End}(W) \text { is a finite morphism. }
$$

Since $W$ is a simple $E$-module it is $G(E)$-simple. On the other hand, $\left.\rho\right|_{H}$ induces a weight decomposition $W=\oplus_{\alpha \in X(H)} W_{\alpha}$, where $H=Z G(E)^{0}$, and each $W_{\alpha}$ is $E$-stable because $H$ is central. Hence, $W=W_{\alpha}$ for some $\alpha \in X(H)$ and $\left.\rho\right|_{H}(t)(w)$ $=\alpha(t) w$ for all $t \in H, w \in W$. Since $\left.\rho\right|_{H}$ is a finite morphism we conclude that $\operatorname{dim} H=1$.

(8.3.3) Corollary. Let $E$ be a reductive, algebraic, monoid with 0 . Then the following are equivalent:

(i) $E$ is g-irreducible.

(ii) $W$ acts transitively on the set of minimal, nonzero idempotents of $Z$.

(iii) There exists a finite morphism $\rho: E \rightarrow \operatorname{End}_{k}(V)$ of algebraic monoids such that $(\rho, V)$ is irreducible as an E-module.

Proof. We have already remarked that (i) is equivalent to (ii), and "(ii) $\Rightarrow$ (iii)" follows from the proof of the lemma. 
Suppose then that we have a morphism $\rho: E \rightarrow \operatorname{End}_{k}(V)$ as in (iii). It suffices to prove (ii) in case $E=\rho(E)$ and $G(E)$ is of adjoint type, since any finite, dominant morphism induces a bijection on the set of $\mathscr{f}$-classes (being a quotient morphism with central kernel). By (3.2), if $e \in I(Z)$ is minimal, then $\chi_{e}: Z \rightarrow k$ is a fundamental generator. On the other hand, if $\lambda$ is the highest weight of $\rho$ with highest weight vector $v$, then $\alpha: Z \rightarrow k=\operatorname{End}_{k}(k v), \alpha(t)(v)=\lambda(t) v$ is actually the character $(\lambda, 1)$ of (3.4). Hence, by (3.5)

$$
\Phi(\rho) \subseteq \text { convex hull of } W \alpha .
$$

But from (3.3) $X(Z)=\langle\Phi(\rho)\rangle$, and thus, $\langle W \alpha\rangle \subseteq X(Z)$ is a finite morphism $\left(\chi^{n} \in\langle W \alpha\rangle\right.$ for all $\chi \in X(Z)$, and some $\left.n=n(x)\right)$ since by $(*) W \alpha$ contains all the extreme points in the rational convex hull of $\Phi(\rho)$. It now follows from (3.1) that $W \alpha=\left\{\chi_{e} \mid e \in I(Z)\right.$ minimal $\}$.

Comment. From the corollary we see that the terminology " $\mathscr{J}$-irreducible" is well chosen. One can further the relationship between minimal $\mathscr{f}$-classes and irreducible representations by embellishing the proof of (8.3.2). The most direct way to get from minimal $\mathscr{Z}$-classes to representations is via the representation on $\mathscr{Z}_{0}(E e)$ (tangent space) induced by left translation of $G(E)$ on $E e$. If $E$ is semisimple and $e$ minimal, then $E e$ is the cone of an ample line bundle on $G / P$, where $P=\{g \in G \mid g e=t e$ for some $\left.t \in Z G^{0}\right\}$. In general $(\operatorname{char}(k)=p>0) \mathscr{Z}_{0}(E e)$ is not irreducible but it is always the dual of some highest weight module of $G(E)$.

Our task now is to interpret the set of all $\mathscr{f}$-irreducible, semisimple monoids as the set of points of a certain rational polyhedral cone associated with the Weyl chamber.

Fix a reductive group $G$ with maximal torus $T$ and Borel subgroup $B \supseteq T$. We assume that $\operatorname{dim} Z G=1$. We adopt the convention that all monoids considered have the same orientation (7.3.1)(b) and we choose to distinguish the polyhedral root systems $(X, \Phi, C)$ and $(X, \Phi, \lambda(C))$ for $\lambda \in \Gamma$ unless $C=\lambda(C)$.

What we wish to classify then (recall (7.3.3)) is the class of pairs $(i, E)$ where $E$ is a semisimple, $\mathscr{F}$-irreducible monoid and $i: G \rightarrow E$ is an isomorphism onto $G(E)$ such that the character $\chi: G \rightarrow k^{*}$ giving the orientation, extends over $i$. The equivalence relation is $\left(i_{1}, E_{1}\right) \sim\left(i_{2}, E_{2}\right)$ if there exists an isomorphism $\alpha: E_{1} \rightarrow E_{2}$ such that $\alpha \circ i_{1}=i_{2}$. The reader who wants "isomorphism classes or nothing" can take $R_{\mathbf{Q}}(G)$ in (8.3.4) and form the orbit set $R_{\mathbf{Q}}(G) / \Gamma$. (Note, however, that $R_{\mathbf{Q}}(G)$ depends only on the isogeny class of $G$ while $\Gamma$ may vary.)

To simplify matters we make use of the fact that given $G$ and $H$ and a central isogeny $j: G \rightarrow H$, we can lift monoids from $H$ to $G$ (using integral closure) and descend them from $G$ to $H$ (using group scheme quotient). Recall (7.1.2) and (7.1.4) for more details.

We can thus assume that $G=G_{0} \times k^{*}$, where $G_{0}$ is semisimple and simply connected. Let $\Delta \subseteq \Phi$ be the base and $\left\{\lambda_{1}, \ldots, \lambda_{r}\right\} \subseteq X_{0}$ the fundamental dominant weights [12, §31.1]. (Here $X_{0}=X\left(T \cap G_{0}\right)$.)

$$
X_{0}^{+}=\left\{x \in X_{0} \mid \chi=\sum_{i=1} m_{i} \lambda_{i}, m_{i} \geqslant 0\right\} .
$$

$\chi \in X=X(T)$, the orientation (7.3.1)(b). Define $\mathscr{E}_{1}(G)=\{(i, E) \mid$ as above $\} / \sim$ and $R_{\mathbf{Q}}(G)=X_{0}^{+} \oplus \mathbf{N} / \sim$ where, in this case $(x, n)>(y, m)$ iff $m x=n y$. 
(8.3.4) THEOREM. There is a canonical bijection $\mathscr{E}_{1}(G) \cong R_{\mathbf{Q}}(G)$.

Proof. Let $(i, E) \in \mathscr{E}_{1}(G)$ and $(X, \Phi, C)$ be its polyhedral root system. By (3.2) and (8.3.3)(ii) $W$ acts transitively on $\left\{\chi_{i}\right\} \subseteq C$. Hence, there exists a unique, fundamental generator $\chi_{1}=\chi+m \in X_{0}^{+} \oplus \mathbf{N} \subseteq X\left(T_{0}\right) \oplus \mathbf{Z}$. Hence, define

$$
(i, E) \rightarrow(\chi, m) \text {. }
$$

Conversely, if $\chi=(\chi, m) \in X^{+} \oplus \mathbf{N}$ represents an element of $R_{\mathbf{Q}}(G)$ let $C_{\lambda}$ be the smallest $W$-stable polyhedral cone containing $(\chi, n)$ (see comment (3) following (3.6)). Then $\left(X, \Phi, C_{\lambda}\right)$ is a polyhedral root system and so determines a bialgebra $A \subseteq k[G]$ as in (7.3.3), and thus a semisimple monoid $E$ with preferred embedding $i$ : $G \rightarrow E$. By construction, $W$ acts transitively on the fundamental generators of $C$. So by (8.3.3), $E$ is $\mathscr{f}$-irreducible. Hence define

$$
\lambda \rightarrow(i, E)=E\left(X, \Phi, C_{\lambda}\right) .
$$

This is well defined and one-to-one because

$$
C_{\lambda}=C_{\lambda^{\prime}}\left\{\begin{array}{l}
\text { iff there exists } k, l>0 \text { such that }(k x, k m)=\left(l x^{\prime}, l m^{\prime}\right) \\
\text { iff } k l m^{\prime} x=k l m x^{\prime} \\
\text { iff } m^{\prime} x=m x^{\prime}
\end{array}\right.
$$

It is a simple matter to check that (1) and (2) are inverses.

(8.4) Smooth monoids. In this final subsection we prove the expected result, that any smooth, semisimple algebraic monoid is isomorphic to $\operatorname{End}_{k}\left(k^{n}\right)$ for some $n$. The proof is now somewhat recreational in the wake of (6.5).

We fix the notation and recall the necessary background that will be used throughout the section.

$E$ is a smooth, semisimple, algebraic monoid with unit group $G$ and maximal $D$-submonoid $Z \subseteq E$. By [27, Theorem 4.4.4] $Z=C_{E}(T)=\{x \in E \mid x t=t x$ for all $t \in T=G(Z)\}$ and by $[8, \S 5], Z$ is a smooth variety. But any smooth affine torus embedding with 0 is isomorphic to $k^{r}$ for some $r[5, \S 3.3]$. Thus, $k[Z]=k\left[\chi_{1}, \ldots, \chi_{r}\right]$, the polynomial algebra on the fundamental generators, and so $\operatorname{Aut}(Z) \cong S_{r}$, the symmetric group on $r$ letters.

Let $(X, \Phi, C)$ be the polyhedral root system of $E$ (with respect to $Z$ ) and let $\Delta \subseteq \Phi$ be a base $R=\left\{\sigma_{\alpha} \in W \mid \alpha \in \Delta\right\}$, where $W$ is the Weyl group. For $1 \leqslant i$, $j-r-1, i \neq j$, let

$$
\sigma_{i j}\left(\chi_{l}\right)= \begin{cases}\chi_{i} & \text { if } l=j \\ \chi_{j} & \text { if } l=i \\ \chi_{l} & \text { if } l \neq i, j\end{cases}
$$

(8.4.1) Lemma. If $\sigma \in R$, then $\sigma=\sigma_{i j}$ for some $i, j, i \neq j$.

Proof. $\sigma \in \operatorname{Aut}(X(T) \otimes \mathbf{R})$ is a (diagonalizable) reflection and $\left\{\chi_{\mathrm{i}}\right\} \subseteq X(T) \otimes \mathbf{R}$ is a $\sigma$-stable basis. Thus, $\sigma$ can interchange only two of the $\chi$ 's since otherwise the eigenspace with eigenvalue -1 will have dimension $\geqslant 2$. 
(8.4.2) LeMma. $W$ acts transitively on $\left\{\chi_{i}\right\}$.

Proof. Since $Z \cong k^{r}$

$$
\operatorname{dim} Z^{W}=\# \text { orbits of } W \text { on }\left\{\chi_{i}\right\} .
$$

But $Z^{W} \subseteq$ center of $G \subseteq E$. Hence, $\operatorname{dim} Z^{W}=1$ since $E$ is semisimple.

(8.4.3) Proposition. $W=S_{r}$.

Proof. Define a graph $\mathrm{Gr}$ as follows: Let $\mathscr{F}=\left\{\chi_{i}\right\}$, the vertices, and $\mathscr{E}=$ $\left\{\left(\chi_{i}, \chi_{j}\right) \in \mathscr{F} \times \mathscr{F} \mid \sigma_{i j} \in R\right\}$, the edges.

Claim. Gr contains no cycles. If it did, we could obtain (upon relabelling):

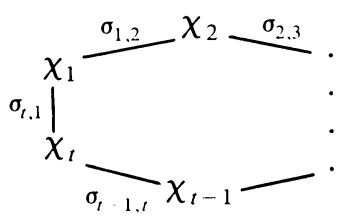

But then $\sigma_{t, 1} \in\left\langle\sigma_{1,2}, \ldots, \sigma_{t-1, t}\right\rangle=S_{t}$, thus contradicting the minimality of $R$ as a generating set of $W[\mathbf{1 2}$, Lemma 29.3C].

So $\mathrm{Gr}$ is a finite graph with $r$ vertices, $r-1$ edges and no cycles. Hence,

$$
1=r-(r-1)=(\text { Euler }) \chi(\mathrm{Gr})=\left|\Pi_{0}(\mathrm{Gr})\right|
$$

and so $\mathrm{Gr}$ is connected. So we can find for each pair $\left(\chi_{i}, \chi_{j}\right)$ a chain

$$
\chi_{i}=\chi_{i, 1}{ }^{\sigma_{1}} \chi_{i, 2}{ }^{\sigma_{2}} \cdots \stackrel{\sigma_{s}}{-} \chi_{i, s}=\chi_{j},
$$

where $\left(\chi_{i, l}, \chi_{i, l+1}\right) \in \mathscr{E}$. But $S_{s+1}=\left\langle\sigma_{1}, \ldots, \sigma_{s}\right\rangle$, and hence $\sigma_{i j} \in W$. Therefore, $W=S_{r}$.

Let $\mathbf{Z}^{r}$ be the free abelian group on $r$ generators with basis $\left\{e_{i}\right\}_{i=1}^{r}$. Recall that " $\langle\cdot, \cdot\rangle$ " denotes " the submonoid generated by".

(8.4.4) Theorem. $(X, \Phi, C) \cong\left(\mathbf{Z}^{r},\left\{e_{i}-e_{j}\right\}_{i \neq j},\left\langle e_{i}\right\rangle\right)$.

Proof. Let $\alpha \in \Phi$ and $\sigma_{\alpha}=\sigma_{i j}$ so that $\sigma_{\alpha}\left(\chi_{i}-\chi_{j}\right)=\chi_{j}-\chi_{i}$. Thus, $\chi_{i}-\chi_{j}=$ $m \alpha$ for some $m \in \mathbf{Z}\left((3.6)\right.$ and (3.7)) since $\chi_{i}-\chi_{j}=\sigma_{\alpha}\left(\chi_{j}\right)-\chi_{j}$.

But $\alpha=\sum c_{i} \chi_{i}$ for some $\left\{c_{i}\right\} \subseteq \mathbf{Z}$ since $\left\{\chi_{i}\right\} \subseteq X$ is a $\mathbf{Z}$-basis, and so $m= \pm 1$ since $\left\{\chi_{i}\right\}$ is also a $\mathbf{Q}$-basis.

Conversely, if we let $\alpha=\chi_{i}-\chi_{j}$, then $\alpha \in \Phi$ since all the $\sigma_{i j}$ 's are conjugate under $S_{r}=W$.

We conclude that $\chi_{i} \rightarrow e_{i}$ induces the desired isomorphism.

(8.4.5) Corollary. $E \cong \operatorname{End}_{k}\left(k^{r}\right)$.

Proof. By (3.8)(2) End ${ }_{k}\left(k^{r}\right)$ has polyhedral root system $\left(\mathbf{Z}^{r},\left\{e_{i}-e_{j}\right\}_{i \neq j},\left\langle e_{i}\right\rangle\right)$, yet by (6.5) any two semisimple monoids with the same polyhedral root systems are isomorphic. So, by (8.4.4), $E \cong \operatorname{End}\left(k^{r}\right)$. 


\section{REFERENCES}

1. M. F. Atiyah and I. G. Mcdonald, Commutative algebra, Addisson-Wesley, Reading Mass., 1969.

2. A. Borel, Algebraic groups, Benjamin, New York, 1969.

3. A. Borel et al., Seminar on algebraic groups and related finite groups, Springer-Verlag, New York, 1970.

4. C. Chevalley, Classification des groupes Lie algébriques, Séminaire École Normale Supérieure, Paris, 1956-1958.

5. V. I. Danilov, The geometry of toric varieties, Russian Math. Surveys (2) 33 (1978), 97-154.

6. M. Demazure, Sous-groupes algébriques de rang maximum du groupe de Cremona, Ann. Sci. École Norm. Sup. 3 (1971), 507-588.

7. M. Demazure and P. Gabriel, Groupes algebriques, North-Holland, Paris, 1970.

8. J. Fogarty, Fixed point schemes, Amer. J. Math. 95 (1973), 35-51.

9. J. A. Green, On the structure of semigroups, Ann. of Math. (2) 54 (1951), 163-172.

10. F. D. Grosshans, Observable groups and Hilbert's fourteenth problem, Amer. J. Math. 95 (1973), 229-253.

11. J. E. Humphreys, Introduction to Lie algebras and representation theory, Springer-Verlag, New York, 1980.

12. Linear algebraic groups, Springer-Verlag, New York, 1981.

13. G. R. Kempf, Instability in invariant theory, Ann. of Math. (2) 108 (1978), 299-316.

14. G. Kempf, F. Knudsen, D. Mumford and B. Saint-Donat, Toroidal embeddings. I. Springer-Verlag, New York, 1973.

15. W. Killing, Die Zusammensetung der Stetigen Endlichen Transformationsgruppen. I-IV, Math. Ann. 31 (1888), 252-290; 33 (1889), 1-48; 34 (1889), 57-122; 36 (1890), 161-189.

16. D. Mumford, Abelian varieties, Oxford Univ. Press, Bombay, 1970.

17. __ Geometric invariant theory, Springer-Verlag, New York, 1982.

18. T. Oda, Torus embeddings and applications, Tata Press, Bombay, 1978.

19. M. S. Putcha, On linear algebraic semigroups, I, II, Trans. Amer. Math. Soc. 259 (1980), 457-469; 471-491.

20. __ On linear algebraic semigroups.III, Internat. J. Math. Math. Sci. 4 (1981), 667-690.

21. Green's relations on a connected algebraic monoid, Linear and Multilinear Algebra 12 (1982), 205-214.

22. __ A semigroup approach to linear algebraic groups, J. Algebra 80 (1983), 164-185.

23. __ Reductive groups and regular semigroups, Semigroup Forum 30 (1984), 253-261.

24. __ Idempotent cross-sections of g-classes, Semigroup Forum 26 (1983), 103-109.

25. On irreducible algebraic monoids, unpublished notes,

26. _ A semigroup approach to linear algebraic groups. II, Roots, J. Pure Appl. Algebra (to appear).

27. L. E. Renner, Algebraic monoids, U. B. C. Thesis, Vancouver, 1982.

28. C_ Cohen-Macaulay algebraic monoids, Proc. Amer. Math. Soc. 89 (1983), 574-578.

29. __ Reductive monoids are von Neumann regular, J. Algebra 93 (1985), 237-245.

30. Classification of semisimple rank one monoids, Trans. Amer. Math. Soc. 287 (1985), 457-473.

31. Classification of semisimple algebraic monoids (unpublished announcement).

32. R. N. Saavedra, Categories tannakiennes, Springer-Verlag, Berlin and New York, 1972.

33. T. A. Springer, Linear algebraic groups, Birkhäuser, Basel, 1981.

34. W. C. Waterhouse, Introduction to affine group schemes, Springer-Verlag, Berlin and New York, 1979.

35. The unit groups of affine algebraic monoids, Proc. Amer. Math. Soc. 85 (1982), 506-508.

Department of Mathematics, York University, Downsview, Ontario, M3J 1P3, Canada

Current address: Department of Mathematics, University of Western Ontario, London, Canada N6A 5B7 